\title{
Sheaf representations and locality of Riesz spaces with order unit
}

\author{
ANTONIO Di NOLA \\ GIACOMO LENZI \\ LUCA SPADA
}

\begin{abstract}
We present an algebraic study of Riesz spaces (ie, real vector lattices) with a (strong) order unit. We exploit a categorical equivalence between those structures and a variety of algebras called RMV-algebras. We prove two different sheaf representations for Riesz spaces with order unit: the first represents them as sheaves of linearly ordered Riesz spaces over a spectral space, the second represents them as sheaves of local Riesz spaces over a compact Hausdorff space. Motivated by the latter representation we study the class of local RMV-algebras. We study the algebraic properties of local RMV-algebra and provide a characterisation of them as special retracts of the real interval $[0,1]$. Finally, we prove that the category of local RMV-algebras is equivalent to the category of all Riesz spaces.
\end{abstract}

2010 Mathematics Subject Classification 00Z99 (primary); 06D35 (secondary)

Keywords: Riesz spaces, sheaf representation, MV-algebras, local algebras.

\section{Introduction}

The algebra of real-valued continuous functions $C(X)$, for $X$ a compact and Hausdorff space, has received great attention in all of its facets (see, eg, Gillman and Jerison [25] and references therein). Indeed, the rich structure of $\mathbb{R}$ lifts to $C(X)$ in several ways, emanating a number of well known structures such as $\mathrm{C}^{*}$-algebras, Banach algebras, vector lattices, etc. In several of those cases the concept of norm is of crucial importance, yet norms elude the classical tools of universal algebra or first order logic.

A purely algebraic and basic structure that can be pulled back on $C(X)$ is the structure of lattice ordered abelian group (for short $\ell$-group). Since $X$ is compact, by Weierstrass' Extreme Value Theorem the functions in $C(X)$ are automatically bounded. This entails that any positively constant function $u$ has the following property:

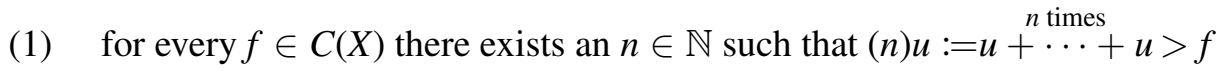


If $G$ is an $\ell$-group, an element $u$ with the property described in Equation (1) is called order unit (or strong order unit). One of the reasons for which the concept of order unit is important is that it allows to define a semi-norm: if $G$ is an $\ell$-group and $g \in G$,

$$
\|g\|:=\inf \left\{\frac{p}{q} \in \mathbb{Q} \mid p, q \in \mathbb{N}, q \neq 0 \text { and }|g|+\cdots+|g| \leq u+\cdots+u\right\}
$$

where $|g|:=g \vee-g$. The operator \|\| is a norm if and only if $G$ is Archimedean. In particular, if $G=C(X)$ then \|\| is the uniform norm (also called sup norm). So, a single element of the algebra allows us to define the norm, but it can be readily seen by a compactness argument that order units are not first-order definable. Nevertheless, as next result shows, this obstruction is only due to the presentation of the structures, so a mere modification of the language dramatically simplifies the situation.

Theorem 1.1 (Mundici [32, Theorem 3.9]) The category of $\ell$-groups with order unit ( $u \ell$-groups, for short) and morphisms preserving the units is equivalent to a class of equationally defined algebras, called MV-algebras.

The importance of Theorem 1.1 cannot be overestimated, in that it allows the study of normed structures with the full paraphernalia of universal algebra.

Recall that a Riesz space is a vector space over the real numbers endowed with an order $\leq$ that is compatible with the vector space operations (ie, such that $x \leq y$ implies $x+z \leq y+z$ and $\lambda x \leq \lambda x$ ) and $\leq$ is a lattice order (ie, every finite subset has a least upper bound and greatest lower bound). The real numbers with their usual operations and order form a Riesz space, also any $L^{p}$-space with the almost everywhere point-wise partial order is a Riesz space. A classical reference for Riesz spaces is Luxemburg and Zaanen [29]. They are important structures in measure theory, but they also have shown to be of interest in economics (see eg Aliprantis and Burkinshaw [1]).

In Di Nola and Leuştean [18] it was noticed for the first time ${ }^{1}$ that the equivalence of Theorem 1.1 can be extended to an equivalence between the category of Riesz spaces with order unit and the category of certain equationally-definable algebras called Riesz MV-algebras (RMV-algebras, for short).

In this work we exploit this equivalence to propose a universal algebraic study of these structures. This approach can be framed in a long list of successful attempts to use the tools of logic in functional analysis as for instance, the theory of approximate truth of

\footnotetext{
${ }^{1}$ The proof in [18] contains a gap, because it relies on a statement by Birkhoff that turned out to be erroneous. We remedy this gap here by giving a self-contained proof of the theorem.
} 
Henson [26], continuous model theory of Chang and Keisler [9], and continuous first order logic of Ben Yaacov and Usvyatsov [3]. (See also the introduction of Iovino [27] for a longer list of examples.)

The plan of the paper is as follows. In Section 2 we recall definitions and basic properties of MV-algebras and RMV-algebras that will be used in the rest of the paper. In Section 3 we give two sheaf representations of RMV-algebras: Corollary 3.11 represents them as sheaves of local RMV-algebras over a compact Hausdorff space; Corollary 3.13 represents them as sheaves of linearly ordered RMV-algebras over a spectral space. In Section 4 we study local RMV-algebras and prove the characterisations summarised below.

Theorem 1.2 Let $A$ be an RMV-algebra. The following are equivalent:

(i) $A$ is local,

(ii) A is isomorphic to an algebra of quasi-constant functions,

(iii) $A$ is generated by its radical,

(iv) $A / \operatorname{Rad}(A) \cong[0,1]$,

(v) $A$ is isomorphic to $\Gamma_{R}(\mathbb{R} \overrightarrow{\times} W,(1,0))$, for some Riesz space $W$, where $\overrightarrow{\times}$ indicates the lexicographic product.

Proof The equivalence of (i) and (ii) is proved in Theorem 4.10. The equivalence of (i) and (iii) is proved in Theorem 4.14. The equivalence of (i) and (iv) is proved in Theorem 4.15. The equivalence of (i) and (v) is proved in Theorem 4.23.

Finally, in Section 4.4 we prove a categorial equivalence between local RMV-algebras and the full category of Riesz spaces-with or without order unit.

\section{Preliminaries}

We briefly recall the definition and some basic properties of MV-algebras needed in the paper, the standard references are Mundici [10] and Mundici [33].

Definition 2.1 An $M V$-algebra is a structure $\left\langle A, \oplus,{ }^{*}, 0\right\rangle$ such that:

(MV 1)

(MV 2)

(MV 3) $\langle A, \oplus, 0\rangle$ is a commutative monoid

$x \oplus 0^{*}=0^{*}$

$\left(x^{*}\right)^{*}=x$ 
(MV 4)

$$
\left(x^{*} \oplus y\right)^{*} \oplus y=\left(y^{*} \oplus x\right)^{*} \oplus x
$$

It is customary to define, in the language of MV-algebras, the following derived operations:

$$
\begin{gathered}
1:=0^{*}, x \odot y:=\left(x^{*} \oplus y^{*}\right)^{*}, x \ominus y:=x \odot y^{*}, \mathrm{~d}(x, y):=(x \ominus y) \oplus(y \ominus x) \\
\text { and for any } n \in \mathbb{N} \quad(n) x:=x \oplus \cdots \oplus x
\end{gathered}
$$

The operation $\mathrm{d}(x, y)$ is called the Chang distance between $x$ and $y$. Every MV-algebra can be endowed with a partial order, by defining:

$$
x \leq y \text { if and only if } x \ominus y=0
$$

In fact the above-defined order always induces a distributive lattice structure where meet and join are term-definable as follows:

$$
x \vee y:=(x \ominus y) \oplus y \text { and } x \wedge y:=\left(x^{*} \vee y^{*}\right)^{*}
$$

Remark 2.2 The operation $\ominus$ and the constant 1 can be equivalently taken as basic operations in the axiomatisation of MV-algebras. The original operations can be recovered by defining $x^{*}:=1 \ominus x$ and $x \oplus y:=\left(x^{*} \ominus y\right)^{*}$.

Example 2.3 The standard MV-algebra is given by the set $[0,1]$, where the operations are interpreted as $x \oplus y=\min (x+y, 1)$ and $x^{*}=1-x$. An easy calculation shows that in this algebra,

$$
\begin{gathered}
x \odot y=\max \{0, x+y-1\}, x \ominus y=\max \{0, x-y\}, \mathrm{d}(x, y)=|x-y|, \\
x \vee y=\max \{x, y\} \text { and } x \wedge y=\min \{x, y\} .
\end{gathered}
$$

As an immediate consequence of Hölder Theorem for ordered groups (see eg Bigard, Keimel, and Wolfenstein $[4,2.6 .3]$ ) one has that the one above is the only structure of MV-algebra that induces the natural order on $[0,1]$, so there is no danger of confusion in speaking of the $M V$-algebra $[0,1]$.

Theorem 2.4 (Chang [8]) The variety of $M V$-algebra is generated, as quasi-variety, by the $M V$-algebra $[0,1]$.

We recall some basic properties of MV-algebras that will be used in the rest of the paper.

Proposition 2.5 In every $M V$-algebra $A$ the following properties hold for every $x, y, z \in A$ : 
(1) if $x=y$ then $x \ominus y=0$

(2) if $x \leq y$ then $x \wedge z \leq y \wedge z$

(3) if $x \wedge y=0$ and $z \wedge y=0$ then $(x \oplus z) \wedge y=0$

(4) if $x \wedge y=0$ then for every $n \in \mathbb{N}, x \wedge(n) y=0$

(5) $x \odot(y \vee z)=(x \odot y) \vee(x \odot z)$

(6) $(x \ominus y) \wedge(y \ominus x)=0$

(7) $\mathrm{d}(x, y) \leq \mathrm{d}(x, z) \oplus \mathrm{d}(z, y)$

(8) $(n) x \vee(n) y=(n)(x \vee y)$

(9) if $x \ominus y=z$ and $x \geq y$ then $y \oplus z=x$

Proof Since all properties from (1) to (9) are quasi-equations, by Theorem 2.4 it is enough to check that they hold in $[0,1]$. We leave to the reader this easy exercise.

We now give the definition of Riesz MV-algebras (RMV-algebras, for short); they are MV-algebras endowed with a scalar multiplication by elements of the real interval $[0,1]$. RMV-algebras were introduced in Di Nola and Leuştean [18] and further studied in Di Nola, Lapenta, and Leuştean [14, 15].

Definition 2.6 (RMV-algebra) A Riesz MV-algebra (RMV-algebra for short) is an $\mathrm{MV}$-algebra $A$ endowed with an external multiplication $f_{r}$ for every real number $r$ in $[0,1]$, satisfying the following conditions. For every $x, y \in A$ and every $r, s \in[0,1]$ :

(RMV 1)

(RMV 2)

(RMV 3)

(RMV 4)

$$
\begin{aligned}
& f_{r}(x \ominus y)=f_{r}(x) \ominus f_{r}(y) \\
& f_{r \ominus s}(x)=f_{r}(x) \ominus f_{s}(x) \\
& f_{r}\left(f_{s}(x)\right)=f_{r \cdot s}(x) \\
& f_{1}(x)=x
\end{aligned}
$$

where $r \cdot s$ indicates the product in $[0,1]$ and $r \ominus s:=\max \{0, r-s\}$. An RMV-algebra is said to be trivial if it satisfies $0=1$.

Note that the MV-algebra [0,1] of Example 2.3 is also an RMV-algebra where $f_{r}(x)=r x$ is the usual multiplication. Similarly to the case of MV-algebras:

Lemma 2.7 The only endomorphism of the RMV-algebra on $[0,1]$ is the identity.

Proof Let $e$ be an endomorphism of the RMV-algebra [0,1]. Since $e$ is a homomorphism, $e(1)=1$, whence for every real $r \in[0,1]$ we have $e(r)=e\left(f_{r}(1)\right)=f_{r}(e(1))=$ $f_{r}(1)=r$. 
Lemma 2.8 (See [18, Lemma 4]) In every RMV-algebra the following properties hold:

(1) $f_{r}(0)=0$

(2) $f_{0}(x)=0$

(3) If $x \leq y$ then $f_{r}(x) \leq f_{r}(y)$

(4) If $r \leq s$ then $f_{r}(x) \leq f_{s}(x)$

(5) $f_{r}(x) \leq x$

Proof (1) For any $r \in[0,1]$,

$$
\begin{array}{rlrl}
f_{r}(0) & =f_{r}(0 \ominus 0) & \text { by Proposition } 2.5(1) \\
& =f_{r}(0) \ominus f_{r}(0) & \text { by (RMV 1) } \\
& =0 & & \text { by Proposition 2.5(1). }
\end{array}
$$

(2) For any $x \in A$,

$$
\begin{aligned}
f_{0}(x) & =f_{r \ominus r}(x) \\
& =f_{r}(x) \ominus f_{r}(x) \\
& =0
\end{aligned}
$$

by Definition 2.6

by (RMV 2)

by Proposition 2.5(1).

(3) If $x \leq y$, then by Equation (5), $x \ominus y=0$, hence, for any $r \in[0,1]$,

$$
\begin{array}{rlr}
0 & =f_{r}(0) & \text { by (1) } \\
& =f_{r}(x \ominus y) & \text { by hypothesis } \\
& =f_{r}(x) \ominus f_{r}(y) & \text { by (RMV 1). }
\end{array}
$$

It follows, again by Equation (5), that $f_{r}(x) \leq f_{r}(y)$.

(4) If $r \leq s$, then by Definition 2.6, $r \ominus s=0$, hence, for any $x \in A$,

$$
\begin{array}{rrr}
0 & =f_{0}(x) & \text { by (2) } \\
& =f_{r \ominus s}(x) & \text { by hypothesis } \\
& =f_{r}(x) \ominus f_{s}(x) & \text { by (RMV 2). }
\end{array}
$$

Hence $f_{r}(x) \leq f_{s}(x)$.

(5) Since for every $r \in[0,1], r \leq 1$, by (4) $f_{r}(x) \leq f_{1}(x)=x$, where the last equality holds because of (RMV 4).

Lemma 2.9 Let $A$ be an RMV-algebra and let $r, s \in[0,1]$ such that $r+s \leq 1$. Then for every $x \in A, f_{(r+s)}(x)=f_{r}(x) \oplus f_{s}(x)$ 
Proof Let $r, s \in[0,1]$ such that $r+s \leq 1$ and $x \in A$. Then

$$
\begin{aligned}
f_{(r+s)}(x) \ominus f_{r}(x) & =f_{(r+s) \ominus r}(x) & & \text { by Equation (RMV 2), } \\
& =f_{s}(x) & & \text { by the definition of } \ominus .
\end{aligned}
$$

Also, by Lemma 2.8(4), $f_{(r+s)}(x) \ominus f_{r}(x)$. So by Proposition 2.5(9) we conclude that $f_{(r+s)}(x)=f_{r}(x) \oplus f_{s}(x)$.

Lemma 2.10 There is only one structure of $R M V$-algebra on $[0,1]$.

Proof As remarked in Example 2.3, there is only one way to endow [0,1] with an MV-algebraic structure, so it only remains to be checked that, in turn, there is only one way to endow this MV-algebra with operations that make it an RMV-algebra. To this end, suppose that for $r \in[0,1]$ the operations $f_{r}$ turn $[0,1]$ into an RMV-algebra. Let $m \in \mathbb{N}$ be such that $m \geq 2$; then by Lemma 2.9, $(m-1) f_{1 / m}(x)=f_{(m-1) / m}(x)$. Hence:

$$
(m-1) f_{1 / m}(x)=f_{(m-1) / m}(x)=f_{1 \ominus 1 / m}(x)=f_{1}(x) \ominus f_{1 / m}(x)=x \ominus f_{1 / m}(x)
$$

So, for every $x \in[0,1]$ the element $f_{1 / m}(x)$ satisfies the equation $(m-1) y=x \ominus y$ in the indeterminate $y$. However, this equation has only one solution in $[0,1]$, given by $x / m$. Therefore it must be that $f_{1 / m}(x)=x / m$. It follows that for every $n<m$, also $f_{n / m}(x)=n x / m$.

Finally, if $r$ is any real number in $[0,1]$ and $m / n \leq r \leq p / q$ then, by Lemma 2.8(4), $f_{m / n}(x) \leq f_{r}(x) \leq f_{p / q}(x)$, so $m x / n \leq f_{r}(x) \leq p x / q$. Letting $m / n$ and $p / q$ tend to $r$, we have $f_{r}(x)=r x$.

In the light of the previous result we will freely refer to $[0,1]$ as an RMV-algebra, for there is no confusion on the RMV-operations defined on it.

Theorem 2.11 Every non-trivial $R M V$-algebra $A$ has a unique $R M V$-subalgebra isomorphic to $[0,1]$, given by the elements of the form $f_{r}(1)$. This subalgebra will henceforth be indicated by $\mathbb{R}(A)$.

Proof We start by noticing that in every RMV-algebra $A$, the subalgebra generated by the empty set is given by:

$$
\mathbb{R}(A):=\left\{f_{r}(1) \mid r \in[0,1]\right\}
$$

Indeed, since 1 is a constant and $f_{r}$ are operations in the language of RMV-algebras, it is clear that those elements belong to the subalgebra of $A$ generated by the empty 
set. The set $\mathbb{R}(A)$ is also a subalgebra of $A$; indeed, by Remark 2.2, it is enough to check that it is closed under the operations $\ominus$ and $f_{r}$ for $r \in[0,1]:$ if $s, t \in[0,1]$, then $f_{s}(1) \ominus f_{t}(1)=f_{s \ominus t}(1)$ by (RMV 2) and $f_{s}\left(f_{t}(1)\right)=f_{s \cdot t}(1)$ by (RMV 4). This ensures that $\mathbb{R}(A)$ is closed under RMV-operations.

Let us now prove that the map that sends $f_{r}(1)$ into $r \in[0,1]$ is well-defined. Let $r \neq s$ be real numbers in $[0,1]$, then either $s<r$ or $r<s$. Assuming without loss of generality that the first inequality holds we obtain $r \ominus s \neq 0$. The axiom RMV 2 gives $f_{r}(1) \ominus f_{s}(1)=f_{r \ominus s}(1)$. We claim that the right-hand side of last equation is different from 0 . Indeed, suppose by way of contradiction that $f_{r \ominus s}(1)=0$. Since $r \ominus s \neq 0$ there exists $m \in \mathbb{N}$ such that $1 / m<r \ominus s$, and by Lemma 2.8(4) this entails $f_{\frac{1}{n}}(1)=0$. Whence, by Lemma $2.9, f_{1}(1)=0$ against the fact that $A$ is non-trivial. So, $f_{r}(1) \ominus f_{s}(1) \neq 0$, therefore by (the contrapositive of) Proposition 2.5(1), we obtain that $f_{r}(1) \neq f_{s}(1)$. It is straightforward to check that this map is an isomorphism of RMV-algebras from $\mathbb{R}(A)$ into $[0,1]$.

Suppose now that $B$ is another subalgebra of $A$ isomorphic to $[0,1]$. Obviously $\mathbb{R}(A)$ is the smallest RMV-subalgebra of $A$, so $\mathbb{R}(A) \subseteq B$. By way of contradiction, suppose there exists $a \in B \backslash \mathbb{R}(A)$. Let $g: \mathbb{R}(A) \rightarrow[0,1]$ and $h: B \rightarrow[0,1]$ be the two given isomorphisms. Set $b:=g^{-1}(h(a)) \in \mathbb{R}(A) \subseteq B$, then in particular $a \neq b$ and both belong to $B$. Now observe that $h(a)=f_{h(a)}(1)$ and thus, by Lemma 2.10,

$$
b=g^{-1}\left(f_{h(a)}(1)\right)=f_{h(a)}\left(g^{-1}\right)(1)=f_{h(a)}\left(1_{\mathbb{R}(A)}\right)
$$

which entails $h(a)=f_{h(a)}(1)=h\left(f_{h(a)}\left(1_{\mathbb{R}(A)}\right)\right)=h(b)$. As $h$ is injective, $a=b$, which contradicts our hypothesis. So $a$ cannot exist and $\mathbb{R}(A)=B$.

Our interest for RMV-algebras stems from the fact that the equivalence of Theorem 1.1 can be extended to an equivalence between RMV-algebras and Riesz spaces with order unit.

Theorem 2.12 There is a functor $\Gamma_{\mathcal{R}}$ from the category of Riesz spaces with order unit to the category of RMV-algebras which is full, faithful and dense, hence it witnesses an equivalence of the aforementioned categories.

This result was originally stated in Di Nola and Leuştean [18]. However, the proof published there is incomplete because it relies on [18, Corollary 2], which is false as in turn it relies on the false Birkhoff [5, Corollary, page 349]. (For a counter-example see Di Nola, Lenzi, Marra, and Spada [16].) We remedy this gap by giving a full proof here. 
Proof of Theorem 2.12 The functor $\Gamma_{\mathcal{R}}$ is defined as follows. If $\langle R,+,-, \cdot, \wedge, \vee, 0, u\rangle \mid$ is a Riesz space with order unit, $\Gamma_{\mathcal{R}}(R, u)$ is defined as the RMV-algebra whose elements are in $[0, u]:=\{r \in R \mid 0 \leq r \leq u\}$ and the operations are defined as:

$$
x \oplus y:=(x+y) \wedge u \quad x^{*}:=u-x \quad f_{r}(x):=r \cdot x
$$

The verification that $\Gamma_{\mathcal{R}}(R, u)$ satisfies axioms (RMV 1)-(RMV 4) is routine. If $h: R \rightarrow S$ is a unit preserving homomorphism of Riesz spaces, $\Gamma_{\mathcal{R}}(h)$ is defined as restriction, ie $\Gamma_{\mathcal{R}}(h):=\left.h\right|_{\Gamma_{\mathcal{R}}(R)}$.

Faithfulness: Let us prove that the functor is faithful, ie it is injective on morphisms. Consider two unit preserving morphisms of Riesz spaces $f, f^{\prime}:(R, u) \rightarrow(S, v)$ which coincide on $[0, u]$ and let $x$ be an arbitrary element of $R$. Since any element of $R$ is the difference of two positive elements, there is no loss of generality is assuming that $x \geq 0$. Then for some integer $n$ we have $0 \leq x \leq n u$. Hence, $0 \leq x / n \leq u$ so, $x / n \in[0, u]$ and $f(x / n)=f^{\prime}(x / n)$, therefore $f(x)=(n) f(x / n)=(n) f^{\prime}(x / n)=f^{\prime}(x)$. We conclude that, $f$ and $f^{\prime}$ coincide on $R$.

Fullness: Let us prove that the functor is full, ie it is surjective on morphisms. Let $\mu$ be a homomorphism of RMV-algebras, then $\mu$ is also a morphism of MV-algebras and, by Mundici [32, Proposition 3.5], it can be lifted to a morphism $\lambda$ of the corresponding $u \ell$-groups $(R, u)$ and $(S, v)$. We prove that $\mu$ is also a Riesz space homomorphism preserving the order unit. Let us write $R^{+}$for the set $\{x \in R \mid x>0\}$ and similarly for $\mathbb{R}$. We preliminary check that the property holds for positive elements, ie if $x \in R^{+}$ and $r \in \mathbb{R}^{+}$, then $\lambda(r x)=r(\lambda x)$. Indeed, under this assumption $x=x_{1}+\ldots+x_{n}$, for suitable $x_{i} \in[0, u]$ and $r=r_{1}+1+\ldots+1$, with $r_{1} \in[0,1]$. Recall that $\lambda$ extends $\mu$, and the latter is an RMV-homomorphism. Hence, $\lambda\left(r_{1} x_{i}\right)=r_{1} \lambda\left(x_{i}\right)$ for every $i \leq n$. From this, by distributivity of scalar multiplication in Riesz spaces, it follows $\lambda(r x)=r(\lambda x)$. Now we can check the general case. Let $x \in R, r \in \mathbb{R}$. Then $x=x_{1}-x_{2}$ where $x_{1}, x_{2} \in R^{+} ; r=r_{1}-r_{2}$, where $r_{1}, r_{2} \in \mathbb{R}^{+}$; and:

$$
\begin{aligned}
\lambda\left(\left(r_{1}-r_{2}\right)\left(x_{1}-x_{2}\right)\right) & =\lambda\left(r_{1} x_{1}+r_{2} x_{2}-r_{1} x_{2}-r_{2} x_{1}\right) \\
& =\lambda\left(r_{1} x_{1}\right)+\lambda\left(r_{2} x_{2}\right)-\lambda\left(r_{1} x_{2}\right)-\lambda\left(r_{2} x_{1}\right) \\
& =r_{1} \lambda\left(x_{1}\right)+r_{2} \lambda\left(x_{2}\right)-r_{1} \lambda\left(x_{2}\right)-r_{2} \lambda\left(x_{1}\right) \\
& =\left(r_{1}-r_{2}\right)\left(\lambda x_{1}-\lambda x_{2}\right) \\
& =\left(r_{1}-r_{2}\right)\left(\lambda\left(x_{1}-x_{2}\right)\right)
\end{aligned}
$$

Density: Finally, we prove that the functor is dense, ie, any RMV-algebra is isomorphic to an algebra in the range of $\Gamma_{\mathcal{R}}$. Let $A$ be a RMV-algebra and let $A^{\prime}$ be its MValgebraic reduct. Since the functor $\Gamma$ of Theorem 1.1, from $u \ell$-groups into MV-algebras, 
is an equivalence of categories, there is a $u \ell$-group $\left(R_{0}, u\right)$ such that $A^{\prime}=\Gamma\left(R_{0}, u\right)$. We extend $R_{0}$ to a Riesz space $R$ such that $\Gamma_{\mathcal{R}}(R, u)=A$. If $s \in \mathbb{R}$, let $z \in \mathbb{Z}$ and $r \in[0,1)$ be such that $s=z+r$, we define, for any $a \in A \subseteq R_{0}$ :

$$
s \cdot a:=a+\cdots+a+f_{r}(a)
$$

By Mundici [10, Chapter 7] every element of $R_{0}$ is a finite sum of elements of $A$ and their opposites, hence the scalar multiplication defined above extends to all $R_{0}$. We leave to the reader the routine checking that $R_{0}$ endowed with this scalar multiplication is a Riesz space. This concludes the proof.

Definition 2.13 (MV and RMV ideals) Let $A$ be an RMV-algebra. A non-empty subset $I$ of $A$ is called $M V$-ideal if $I$ is downward closed (ie, $x \leq y$ and $y \in I$ imply $x \in I$ ) and it is closed under $\oplus$. A non-empty subset $J$ of $A$ is called $R M V$-ideal if it is an MV-ideal and it is closed under the operations $f_{r}$, ie, for every $r \in[0,1]$, if $x \in J$ then $f_{r}(x) \in J$.

Lemma 2.14 For any RMV-algebra $A$, there is an isomorphism from the lattice of $M V$-congruences of $A$ and the lattice of its MV-ideals. Similarly, there is a lattice isomorphism between $R M V$-congruences of $A$ and its $R M V$-ideals.

Proof Both isomorphisms are defined in the same way: if $J$ is an MV- or RMV-ideal and $\theta$ is an MV- or RMV-congruence of A then:

$$
\theta \mapsto J_{\theta}:=\{a \in A \mid a \theta 0\} \quad J \mapsto \theta_{J}:=\left\{(a, b) \in A^{2} \mid \mathrm{d}(a, b) \in J\right\}
$$

The fact that they are isomorphisms is well-known in the case of MV-algebras (see eg [10, Proposition 1.2.6]); the case of RMV-algebra is a straightforward adaptation of the argument for MV-algebras. See also Di Nola, Lenzi, Marra, and Spada [16] for a general proof that has both MV and RMV as special cases.

Lemma 2.15 In any RMV-algebra $A, M V$-ideals and $R M V$-ideals coincide. Therefore $M V$-congruences and $R M V$-congruences coincide.

Proof By definition, every RMV-ideal is an MV-ideal. Vice versa, let $A$ be an RMV-algebra and $J \subseteq A$ be an MV-ideal. By Lemma 2.8(5), $f_{r}(x) \leq x$ for every $x \in A$ and for every $r \in[0,1]$, hence $J$ is an RMV-ideal.

In the light of the previous lemma, we will simply speak of ideals. 
Notation If $A$ is an RMV-algebra, $J$ is an ideal of $A$ and $a \in A$, we henceforth write $[a]_{J}$ for the image of $a$ under the natural epimorphism induced by the congruence $\theta_{J}$. If $S \subseteq A$, we write $\langle S\rangle$ for the ideal generated by the set $S$, ie the smallest ideal containing $S$. Finally, If $I$ and $J$ are ideals, we write $I \vee J$ for the ideal generated by the set $I \cup J$.

Lemma 2.16 (See [10, Lemma 1.2.1]) Let $A$ be an $R M V$-algebra and $x, y \in A$. Then $x \in\langle\{y\}\rangle$ if and only if there exists $n \in \mathbb{N}$ such that $x \leq(n) y$.

The following is a folklore result for MV-algebras. For sake of completeness we give here a proof for RMV-algebras.

Lemma 2.17 Let $A$ be an RMV-algebra and $a, b \in A$. Let $\langle\{a\}\rangle,\langle\{b\}\rangle$ be the principal ideals generated by $a$ and $b$, respectively. Then $\langle\{a\}\rangle \cap\langle\{b\}\rangle=\langle\{a \wedge b\}\rangle$.

$$
\begin{array}{rlr}
\text { Proof } x \in\langle\{a\}\rangle \cap\langle\{b\}\rangle & \\
& \Leftrightarrow \quad \exists n \in \mathbb{N} \text { s.t. } x \leq(n) a \text { and } x \leq(n) b & \text { by Lemma } 2.16 \\
& \Leftrightarrow \quad \exists n \in \mathbb{N} \text { s.t. } x \leq(n) a \wedge(n) b & \\
& \Leftrightarrow \quad \exists n \in \mathbb{N} \text { s.t. } x \leq(n)(a \wedge b) & \text { by Proposition 2.5(8) } \\
& \Leftrightarrow \quad x \in\langle\{a \wedge b\}\rangle & \text { by Lemma 2.16. }
\end{array}
$$

Definition 2.18 (Prime, maximal and primary ideals) Let $A$ be RMV-algebra. An ideal $I$ of $A$ is called

(1) maximal if it is maximal among proper ideals with respect to the inclusion order-we call $\operatorname{Max}(A)$ the set of all maximal ideals of $A$;

(2) prime if $x \wedge y \in I$ implies that either $x \in I$ or $y \in I$, we call $\operatorname{Spec}(A)$ the set of all prime ideals of $A$; and

(3) primary if it is contained in a unique maximal ideal, we call $\operatorname{Prim}(A)$ the set of all primary ideals of $A$.

Definition 2.19 (Radical) The intersection of all maximal ideals of an RMV-algebra $A$ is called the radical of $A$ and denoted by $\operatorname{Rad}(A)$. We call the elements of $\operatorname{Rad}(A)$ infinitesimals.

Definition 2.20 (Local RMV-algebras) A non-trivial RMV-algebra $A$ is called local if it contains a unique maximal ideal (or, equivalently, if $\operatorname{Rad}(A)$ is a maximal ideal).

We postpone to Section 4.1 a discussion on the aptness of the attribute local in the above definition. 
Remark 2.21 Notice that local RMV-algebras do not form a subvariety, in fact no proper non-trivial subvariety of RMV-algebras exists as shown in Corollary 2.30 below. However, the class of local RMV-algebras is axiomatised, within the variety of RMV-algebras, by the first order formula:

$$
\forall x\left(x \leq x^{*} \text { OR } x^{*} \leq x \text { OR } \mathrm{d}\left(x, x^{*}\right)^{2}=0\right)
$$

Indeed, an RMV-algebra is local if and only if its MV-algebra reduct is local and the above formula is known to axiomatise local MV-algebras Di Nola, Esposito, and Gerla [13, Theorem 8.1].

Lemma 2.22 Let $A$ be $R M V$-algebra and I be an ideal of $A$, then:

(1) $I$ is maximal if and only if $A / I$ is isomorphic to [0,1], in a unique way.

(2) $I$ is prime if and only if the lattice order of $A / I$ is linear.

(3) I is primary if and only if $A / I$ is local.

Proof (1) The quotient of an MV-algebra by a maximal ideal is isomorphic (in a unique way) to a subalgebra of [0,1] (see eg Marra and Spada [31, Lemma 3.8]). As shown in the proof of Theorem 2.11, any non-trivial 0-generated RMV-subalgebra is isomorphic to $[0,1]$, so the quotient of an RMV-algebra by a maximal ideal must be isomorphic to $[0,1]$.

(2) In an MV-algebra this statement holds (see eg Mundici [33, Proposition 4.13]). Hence, by Lemma 2.15 the statement is also true for RMV-algebras.

(3) Notice that the natural quotient map $\pi_{I}: A \rightarrow A / I$ gives an isomorphism between the lattice of ideals of $A$ containing $I$ and the lattice of ideals of $A / I$ (see eg Burris and Sankappanavar [6, Theorem 6.20, Chapter II]). Now suppose that $I$ is primary and let $M$ be the unique maximal ideal containing $I$. Then $M / I$ must be the unique maximal ideal of $A / I$, so $A / I$ is local. Conversely, suppose that $A / I$ is local and let $N, N^{\prime}$ be maximal ideals of $A$ containing $I$. Then $N / I$ and $N^{\prime} / I$ are maximal ideals of $A / I$. Since $A / I$ is local, we have $N / I=N^{\prime} / I$. Since $N$ and $N^{\prime}$ both contain $I$, this implies $N=N^{\prime}$. So $I$ is primary.

We now recall a few results on RMV-algebras which are either already known or easy consequences of the fact that they hold in MV-algebras.

Definition 2.23 Let $A$ be an RMV-algebra and $P \subseteq A$, we define

$$
\begin{gathered}
O(P):=\{x \in A \mid x \wedge y=0 \text { for some } y \notin P\} \\
P^{\perp}:=\{x \in A \mid x \wedge y=0 \text { for all } y \in P\}
\end{gathered}
$$

We write $y^{\perp}$ as a shorthand for $\{y\}^{\perp}$. 
Lemma 2.24 Let A be RMV-algebra. The following hold.

(1) If $P \subseteq A$, then $O(P)=\bigcup\left\{y^{\perp} \mid y \notin P\right\}$.

(2) If $P \in \operatorname{Spec}(A)$, then $O(P)=\bigcap\{M \in \operatorname{Spec}(A) \mid M$ is minimal in the order $\subseteq$ and $M \subseteq P\}$.

(3) If $P \in \operatorname{Spec}(A)$, then $O(P)$ is a primary ideal contained in $P$.

(4) If $I$ is an ideal of $A$, then $I^{\perp}=\bigcap\{P \in \operatorname{Spec}(A) \mid I \nsubseteq P\}$.

(5) $y^{\perp}=(\langle\{y\}\rangle)^{\perp}$

(6) If $I \subseteq A$, then $I^{\perp}$ is an ideal of $A$.

(7) If $H=I^{\perp}$ then $H \cap I=\{0\}$.

(8) Let $H$ and $I$ be ideals of $A$, if $H \cap I=\{0\}$ then $H \subseteq I^{\perp}$.

Proof For (1), $x \in O(P)$ if and only if there exists $y \notin P$ such that $x \wedge y=0$. This in turn is equivalent to $\exists y \notin P$ such that $x \in y^{\perp}$, in other words $x \in \bigcup\left\{y^{\perp} \mid y \notin P\right\}$.

(2) to (4) are proved for MV-algebras in Ferraioli and Lettieri [21, Proposition 2.10], Filipoiu and Georgescu [22, Lemma 2.1 and 2.4] and [21, Lemma 2.7], respectively. In the light of Lemma 2.15 the results hold also for RMV-algebras.

One inclusion in (5) is obvious. For the other, suppose $x \in y^{\perp}$, ie $x \wedge y=0$. Let $z \in\langle\{y\}\rangle$, then by Lemma 2.16, there exists $n \in \mathbb{N}$ such that $z \leq(n) y$. By Proposition 2.5(2) $x \wedge z \leq x \wedge(n) y$ and the latter is equal to 0 by (4) of the same proposition, so $x \wedge z=0$, whence $x \in(\langle\{y\}\rangle)^{\perp}$.

For (6), let $I \subseteq A$ and take $x, y \in I^{\perp}$, hence for all $z \in I$ we have $x \wedge z=y \wedge z=0$, by Proposition 2.5(3) we also have $(x \oplus y) \wedge z=0$, hence $I^{\perp}$ is closed under $\oplus$. The fact that $I^{\perp}$ is downward closed is an immediate consequence of the monotonicity of $\wedge$, Proposition 2.5(2).

To prove (7), suppose $h \in H$, then by hypothesis $h \wedge i=0$ for all $i \in I$. So, if $h$ also belongs to $I$, then $h \wedge h=0$, whence $h=0$.

To prove (8) we reason by contradiction. Suppose that there exists $h \in H$ and $h \notin I^{\perp}$; the latter implies that there exists some $i \in I$ such that $h \wedge i \neq 0$, but $H$ and $I$ are downward closed, so $h \wedge i$ belongs to both of them and is different from 0 .

Definition 2.25 Henceforth we will indicate by $\operatorname{Prim}_{0}(A)$ the set of primary ideals of an RMV-algebra $A$ that have the form $O(M)$ for some $M \in \operatorname{Max}(A)$.

Lemma 2.26 In every $R M V$-algebra $A$ :

$$
\bigcap_{I \in \operatorname{Prim}(A)} I=\{0\}
$$


As a consequence, every $R M V$-algebra is isomorphic to a subdirect product of local RMV-algebras.

Proof The first statement was originally proved for MV-algebras in [22, Lemma 2.2]. In the light of Lemma 2.15 the results holds also for RMV-algebras. The second statement is an exercise in universal algebra, combined with Lemma 2.22(3).

Lemma 2.26 has an analogue in terms of linearly ordered RMV-algebras.

Lemma 2.27 Every RMV-algebra is isomorphic to the subdirect product of linearly ordered $R M V$-algebras.

Proof This is known as Chang's subdirect representation theorem for MV-algebras [10, Theorem 1.3.3], which asserts that subdirectly irreducible MV-algebras are linearly ordered. In the light of Lemma 2.15 the same holds for RMV-algebras.

Lemma 2.28 (Chinese reminder theorem for RMV-algebras) Let $I_{1}, I_{2}, \ldots, I_{n}$ be ideals of an $R M V$-algebra $A$ and let $a_{1}, a_{2}, \ldots, a_{n}$ be elements of $A$ such that $\left[a_{i}\right]_{I_{i} \vee I_{j}}=\left[a_{j}\right]_{I_{i} \vee I_{j}}$ for $i, j=1,2, \ldots, n$. Then there exists $a \in A$ such that $[a]_{I_{i}}=\left[a_{i}\right]_{I_{i}}$ for $i=1,2, \ldots, n$.

Proof This was originally proved for MV-algebras in [21, Theorem 2.6]. In the light of Lemma 2.15 the results holds also for RMV-algebras.

Theorem 2.29 (Marchioni [30, Theorem 3.5]) Every linearly ordered RMV-algebra $A$ can be embedded in a suitable ultrapower of the $R M V$-algebra $[0,1]$ that depends only on the cardinality of $A$. As a consequence, every RMV-algebra $B$ is isomorphic to an $R M V$-algebra of functions from $\operatorname{Spec}(B)$ into a suitable ultrapower $[0,1]^{*}$, by the map that sends any $b \in B$ into the function $g_{b}: \operatorname{Spec}(B) \rightarrow[0,1]^{*}$ such that $g_{b}(P)=[b]_{P}$.

Corollary 2.30 No proper non-trivial sub-quasi-variety of RMV-algebra exists (a fortiori, no proper non-trivial subvariety of $R M V$-algebras exists).

Proof By Theorem 2.11, every non-trivial RMV-algebra contains a copy of $[0,1]$, which, by Theorem 2.29 generates the full class of all RMV-algebras as a quasivariety. 


\section{Sheaf representation}

Several sheaf representations for MV-algebras are known, we refer to Gehrke, van Gool, and Marra [24] for a complete and unifying account of them. In this section we provide two sheaf representations of RMV-algebras: Corollary 3.11 and Corollary 3.13. When combined with Theorem 2.12, the two results afford two sheaf representations of Riesz spaces with order unit.

The first result is similar to the representation proved for MV-algebras in Filipoiu and Georgescu [22]. However, the proof we present here is much shorter as it uses a general and powerful criterion in Davey [12]. The second result is similar to the representation proved for MV-algebras in Dubuc and Poveda [19]. Also in this case our proof is much shorter as it uses a general result in Cornish [11].

We recall a few basic definitions and some results from [12], for the sake of completeness and to fix the notation.

Definition 3.1 (Sheaf space) A sheaf space of sets is a triple $(F, X, \pi)$ where $X$-the base space - and $F$ - the étalé space - are topological spaces, and $\pi: F \rightarrow X$ is a local homeomorphism, ie, for each $a \in F$, there exist open sets $A \ni a$ and $A^{\prime} \ni \pi(a)$, such that $\pi$ is a homeomorphism from $A$ into $A^{\prime}$.

If $x \in X$, we denote by $F_{x}$ the set $\pi^{-1}(x)$ and we call it the stalk at $x$. If $Y \subseteq X$, then $F(Y)$ denotes the set of continuous maps $\sigma: Y \rightarrow F$, such that $\sigma(y) \in F_{y}$ for all $y \in Y$. The elements of $F(Y)$ are called (local) sections over $Y$, the elements of $F(X)$ are called global sections.

Definition 3.2 (Sheaf space of algebras) Let $\mathcal{L}$ be a purely functional first order language. A sheaf space of sets $(F, X, \pi)$ is called sheaf of algebras of type $\mathcal{L}$ if

(1) for each $x \in X, F_{x}$ is an algebra of type $\mathcal{L}$, and

(2) for each open subset $U$ of $X$ the set $F(U)$ forms an algebra of type $\mathcal{L}$ under point-wise operations.

Let $\mathbb{K}$ be a class of algebras in some language $\mathcal{L}$, if $(F, X, \pi)$ is a sheaf space of algebras of type $\mathcal{L}$ such that conditions in (1) and (2) above hold with "algebra of type $\mathcal{L}$ " replaced by "algebra in $\mathbb{K}$ ", then $(F, X, \pi)$ is a sheaf space of $\mathbb{K}$-algebras.

Remark 3.3 If in the above definition $\mathbb{K}$ is a variety (=equational class of algebras), then the conditions of (1) and (2) are equivalent (see [12, Lemma 1.2] and the ensuing discussion). 
It is shown in [12, Theorem 1.5] that, in the context of varieties, the definition above of sheaf space of $\mathbb{K}$-algebras is equivalent to the classical definition of sheaves as contravariant functors with values in $\mathbb{K}$ satisfying the gluing axioms.

\subsection{Compact sheaf representation}

Given an algebra $A$, an element $a \in A$, and a congruence $\Theta$ on $A$, we indicate by $[a]_{\Theta}$ the equivalence class of $a$ in $A / \Theta$. If $\left\{\Theta_{x} \mid x \in X\right\}$ is a family of congruences on an algebra $A$ and $U \subseteq X$, we define $\Theta_{U}=\bigcap_{x \in U} \Theta_{x}$. Since we will often work with ideals rather than congruences, if $I$ is an ideal of $A$ and $a \in A$, we will write $[a]_{I}$ for $[a]_{\theta_{I}}$, where $\theta_{I}$ is the congruence associated to $I$ by Lemma 2.14 .

Definition 3.4 (The sheaf associated to an algebra) Given an algebra $A$, a topological space $(X, \tau)$, and a family $\left\{\Theta_{x} \mid x \in X\right\}$ of congruences on $A$, one can define the triple $\left(F_{A}, X, \pi\right)$ as follows:

i) $F_{A}$ is the disjoint union of the quotients $A / \Theta_{x}$ as $x$ varies in $X$; in symbols:

$$
F_{A}:=\biguplus_{x \in X} A / \Theta_{x}
$$

ii) The function $\pi$ is defined as the projection from $F_{A}$ into $X$, ie, $\pi\left([a]_{\Theta_{x}}\right):=x$.

iii) Upon defining, for any $a \in A$, the function $\hat{a}: X \rightarrow F_{A}$ as $\hat{a}(x):=[a]_{\Theta_{x}}$, the set $F_{A}$ is endowed with the topology generated by the sub-basis $\{\hat{a}[U] \mid U \in \tau, a \in A\}$.

In general, the above-defined triple may fail to be a sheaf of algebras. The following result, due to Davey, characterises in terms of the topology on $X$ the cases in which the triple is indeed a sheaf .

Theorem 3.5 ([12, Lemma 2.1, Corollary 2.2]) Let $A$ be an algebra in a language $\mathcal{L}$. Let $\left\{\Theta_{x} \mid x \in X\right\}$ be a family of congruences on $A$ and $\tau$ be a topology on $X$.

(1) The triple defined in Definition 3.4 is a sheaf of $\mathcal{L}$-algebras if and only if the following condition holds:

For any $a, b \in A$ and any $x \in X$, if $[a]_{\Theta_{x}}=[b]_{\Theta_{x}}$, then there exists an open set $U \ni x$ such that $[a]_{\Theta_{U}}=[b]_{\Theta_{U}}$,

where $\Theta_{U}:=\bigcap_{x \in U} \Theta_{x}$.

(2) Additionally, if the above condition holds, and $A$ is a subdirect product of the algebras $\left\{A / \Theta_{x} \mid x \in X\right\}$, then the assignment $a \mapsto \hat{a}$ from the algebra $A$ into the algebra of global sections of $\left(F_{A}, X, \pi\right)$ is an injective $\mathcal{L}$-homomorphism. 
The set of maximal ideals of an MV-algebra $A$ is usually regarded as a topological space by endowing it with the Zariski-like topology generated by the open sets of the form $\{M \in \operatorname{Max}(A) \mid a \notin M\}$ for $a \in A$. The problem is that in general maximal ideals are not enough to separate the elements of an arbitrary RMV-algebra. So, Theorem 3.5 would not necessarily provide an embedding if applied to the set of congruences corresponding to maximal ideals. In view of Lemma 2.26 we may remedy this by considering the obvious bijection $O: \operatorname{Max}(A) \rightarrow \operatorname{Prim}_{0}(A)$, that sends $M \in \operatorname{Max}(A)$ into $O(M)$. Indeed, the map is clearly surjective, and it is injective because every primary ideal is contained in a unique maximal ideal. Using this bijection we endow $\operatorname{Prim}_{0}(A)$ with a topology that makes it homeomorphic to $\operatorname{Max}(A)$, as follows.

Definition 3.6 We endow the set $\operatorname{Prim}_{0}(A)$ with the topology generated by the following open sets:

$$
U(a):=\left\{O(M) \in \operatorname{Prim}_{0}(A) \mid a \notin M\right\} \text { for } a \in A
$$

Remark 3.7 It is an immediate consequence of the Definition 3.6 that the space $\operatorname{Max}(A)$ is homeomorphic to $\operatorname{Prim}_{0}(A)$.

Theorem 3.8 Let $A$ be an RMV-algebra. Consider the family of congruences $\left\{\theta_{P} \mid P \in \operatorname{Prim}_{0}(A)\right\}$, where $\theta_{P}$ is the congruence in bijection, according to Lemma 2.14, with $P \in \operatorname{Prim}_{0}(A)$. Then $\left(F_{A}, \operatorname{Prim}_{0}(A), \pi\right)$ constructed as in Definition 3.4 is a sheaf of local RMV-algebras over a compact Hausdorff space and $A$ embeds into the algebra of global sections of $\left(F_{A}, \operatorname{Prim}_{0}(A), \pi\right)$ by the assignment that sends $a \in A$ into $\hat{a} \in F_{A}\left(\operatorname{Prim}_{0}(A)\right)$.

Proof In order to prove that $\left(F_{A}, \operatorname{Prim}(A), \pi\right)$ is a sheaf of algebras we use Theorem 3.5(1). To this end we first need to prove:

Claim 1 For any $a, b \in A$, the set $U:=\left\{Q \in \operatorname{Prim}_{0}(A) \mid[a]_{Q}=[b]_{Q}\right\}$ is open in the topology defined in (9).

Proof of Claim 1 Let $Q \in \operatorname{Prim}_{0}(A)$ and $M$ be the unique maximal ideal for which $Q=O(M)$. Notice preliminarily that by Lemma 2.14 if $Q \in U$, then $\mathrm{d}(a, b) \in Q$. By definition of $O(M)$ there exists $c \notin M$ such that $\mathrm{d}(a, b) \wedge c=0$. Since $Q=O(M)$, we have $Q \in U(c)$. In addition $U(c) \subseteq U$. Indeed, if $R \in U(c)$, let $N$ be the maximal ideal such that $R=O(N)$, then by definition of $U(c), c \notin N$ and since $\mathrm{d}(a, b) \wedge c=0$ the definition of $O(N)$ entails that $\mathrm{d}(a, b) \in O(N)=R$ whence $[a]_{R}=[b]_{R}$. We conclude that $U$ can be written as the union of all $U(c)$ where each $c \in A$ is found as above, hence $U$ is open. 
As a consequence of Claim 1, the main condition of Theorem 3.5 is satisfied. Indeed, Let $a, b \in A$ and fix any $P \in \operatorname{Prim}_{0}(A)$, suppose that $[a]_{P}=[b]_{P}$, then by Claim 1 the set $U$ is open, by its definition $[a]_{U}:=[a]_{\bigcap_{P \in U} P}=[b]_{\bigcap_{P \in U} P}=[b]_{U}$, and obviously $P \in U$. By Theorem 3.5(1) we conclude that $\left(F_{A}, \operatorname{Prim}_{0}(A), \pi\right)$ is a sheaf of RMV-algebras.

The stalks $\left(F_{A}\right)_{P}$ for $P \in \operatorname{Prim}_{0}(A)$ are local by Lemma 2.22(3) and Lemma 2.24, for $\left(F_{A}\right)_{P} \cong A / P$. By Remark $3.7 \operatorname{Prim}_{0}(A)$ is homeomorphic to $\operatorname{Max}(A)$; since the latter is known to be compact and Hausdorff (see eg Cabrer and Spada [7, Remark 3.8]) $\operatorname{Prim}_{0}(A)$ will be as well.

Finally, by Lemma 2.26, the ideals in $\operatorname{Prim}_{0}(A)$ are enough to afford a subdirect decomposition of $A$, so by Theorem 3.5 the assignment $a \mapsto \hat{a}$ is an injective RMVhomomorphism.

Remark 3.9 Notice that any open set in $\operatorname{Prim}_{0}(A)$ with the topology given in (9) can be written in the form $U(I):=\{O(M) \mid I \nsubseteq M\}$, with $I$ a suitable ideal of $A$. Indeed if $U$ is open, then it can be written as $U=\bigcup_{a \in S} U_{a}$ for some subset $S$ of $A$. Then if we set $I:=\langle S\rangle$, we have that if $O(M) \in U$, then there exists $a \in S$ such that $a \notin M$, so also $\langle\{a\}\rangle \nsubseteq M$, whence $I \nsubseteq M$. Vice versa, if $I \nsubseteq M$, then $S \nsubseteq M$, whence there exists $a \in S$ such that $a \notin M$.

Lemma 3.10 The assignment $a \mapsto \hat{a}$ of Theorem 3.8 is surjective; in other words for every global section $\sigma \in F_{A}\left(\operatorname{Prim}_{0}(A)\right)$ there exists $a \in A$ such that $\sigma=\hat{a}$.

Proof The proof is similar to Ferraioli [20, Proposition 4.1.5]; we spell out the details for the sake of completeness. Let $I$ be an index set such that $\left\{P_{i} \mid i \in I\right\}=\operatorname{Prim}_{0}(A)$. Fix $P_{i} \in \operatorname{Prim}_{0}(A)$, by Definition 3.1 $\sigma\left(P_{i}\right) \in A / P_{i}$, so there exists $a_{i} \in A$ such that $\sigma\left(P_{i}\right)=\left[a_{i}\right]_{P_{i}}$, hence $\sigma\left(P_{i}\right)=\hat{a}_{i}\left(P_{i}\right)$. By Theorem 3.5(1), there is an open set $U_{i}$ containing $P_{i}$, such that:

$$
\sigma(Q)=\hat{a}(Q), \text { for any } Q \in U_{i}
$$

There is no loss of generality in taking $U_{i}$ to be a basic open, so there exists $b_{i} \in A$ such that $U_{i}=U\left(b_{i}\right)$. The open sets $\left\{U\left(b_{i}\right) \mid i \in I\right\}$ form an open covering of $\operatorname{Prim}_{0}(A)$ so, by compactness a finite open covering $\left\{U\left(b_{1}\right), \ldots, U\left(b_{n}\right)\right\}$ can be extracted. Since

$$
\operatorname{Prim}_{0}(A)=\bigcup_{i=1}^{n} U\left(b_{i}\right)=U\left(b_{1} \vee \cdots \vee b_{n}\right)
$$

one has that $\left\langle\left\{b_{1}\right\}\right\rangle \vee \cdots \vee\left\langle\left\{b_{n}\right\}\right\rangle=A$. Let us define:

$$
H_{i j}:= \begin{cases}A & \text { if } U\left(b_{i} \wedge b_{j}\right)=\emptyset \\ \bigcap\left\{Q \in \operatorname{Prim}_{0}(A) \mid Q \in U\left(b_{i} \wedge b_{j}\right)\right\} & \text { otherwise }\end{cases}
$$


Notice that if $Q \in U\left(b_{i} \wedge b_{j}\right)$ then $Q \in U\left(b_{i}\right) \cap U\left(b_{j}\right)$, so by (10), $\sigma(Q)=\left[a_{i}\right]_{Q}=\left[a_{j}\right]_{Q}$, hence $\left[a_{i}\right]_{H_{i j}}=\left[a_{j}\right]_{H_{i j}}$ for any $i, j \leq n$. For any $i, j, h \leq n$, by Proposition 2.5(7) $\mathrm{d}\left(a_{i}, a_{j}\right) \leq \mathrm{d}\left(a_{i}, a_{h}\right) \oplus \mathrm{d}\left(a_{h}, a_{j}\right) \in H_{i h} \vee H_{h j}$. So, letting $I_{i}:=\left\langle\left\{b_{i}\right\}\right\rangle$, the ideal generated by $b_{i}$ :

$$
\begin{aligned}
\mathrm{d}\left(a_{i}, a_{j}\right) \in \bigcap_{h=1}^{n}\left(H_{i h} \vee H_{h j}\right) & =\left(\bigcap_{h=1}^{n}\left(H_{i h} \vee H_{h j}\right)\right) \cap \bigvee_{k=1}^{n} I_{k} \quad \text { because } \bigvee_{k=1}^{n} I_{k}=A \\
& =\bigvee_{k=1}^{n}\left(\bigcap_{h=1}^{n}\left(H_{i h} \vee H_{h j}\right) \cap I_{k}\right) \\
& \subseteq \bigvee_{k=1}^{n}\left(\left(H_{i k} \vee H_{k j}\right) \cap I_{k}\right) \\
& =\bigvee_{k=1}^{n}\left(H_{i k} \cap I_{k}\right) \vee \bigvee_{k=1}^{n}\left(H_{k j} \cap I_{k}\right)
\end{aligned}
$$

Let $J_{i}:=\bigvee_{k=1}^{n}\left(H_{i k} \cap I_{k}\right)$. Then $\left[a_{i}\right]_{J_{I} \vee J_{j}}=\left[a_{j}\right]_{J_{i} \vee J_{j}}$ for $i, j=1,2, \ldots, n$. So, by the Chinese Remainder Theorem (Lemma 2.28) there exists $a \in A$ such that $[a]_{J_{i}}=\left[a_{i}\right]_{J_{i}}$ for $i=1,2, \ldots, n$. In other words, for every $i \leq n$, we have $\mathrm{d}\left(a_{i}, a\right) \in J_{i}$. As the next step we claim that

Claim 2 For any $i, k \leq n, H_{i k} \cap I_{k} \subseteq I_{i}^{\perp}$.

Proof of Claim 2 We reason by cases.

If $U\left(b_{i} \wedge b_{k}\right)=\emptyset$, then by Lemma $2.26 b_{i} \wedge b_{k}=0$, it follows by Lemma 2.24(5) that $b_{k} \in I_{i}^{\perp}$, hence, using Lemma 2.24(6), we have $I_{k} \subseteq I_{i}^{\perp}$. Furthermore, since in this case by definition $H_{i k}=A$, it holds $H_{i k} \cap I_{k}=I_{k}$. So we conclude $H_{i k} \cap I_{k} \subseteq I_{i}^{\perp}$.

If $U\left(b_{i} \wedge b_{k}\right) \neq \emptyset$, then by definition $H_{i k}=\bigcap\left\{O(N) \mid N \in U\left(b_{i} \wedge b_{k}\right)\right\}=\bigcap\{O(N) \mid$ $\left.N \in U\left(\left\langle\left\{b_{i} \wedge b_{k}\right\}\right\rangle\right)\right\}=\bigcap\left\{O(N) \mid N \in U\left(I_{i} \cap I_{k}\right)\right\}$, where the latter equality holds because of Lemma 2.17. By way of contradiction suppose that $x \in H_{i k} \cap I_{k}$ but $x \notin I_{i}^{\perp}$. This means that there exists $a \in I_{i}$ such that $x \wedge a \neq 0$. By Lemma 2.26 there exists $N \in \operatorname{Prim}_{0}(A)$ such that $x \wedge a \notin N$. But $x \wedge a \in I_{i} \cap I_{k}$, because $x \in I_{k}$ and $a \in I_{i}$ and they are both downward closed. So, $N \in U\left(I_{i} \cap I_{k}\right)$ and since $x \in H_{i k}$, we have $x \in N$. In turn this would imply $x \wedge a \in N$, which contradicts our hypothesis. So, in both cases we have $H_{i k} \cap I_{k} \subseteq I_{i}^{\perp}$.

As an immediate consequence of the claim and Lemma 2.24(6) we obtain that $J_{i} \subseteq I_{i}^{\perp}$ for every $i \leq n$. In addition $I_{i}^{\perp} \subseteq O(N)$ for every $N \in U\left(b_{i}\right)$, because if $N \in U\left(b_{i}\right)$, 
then $b_{i} \notin N$, hence byLemma 2.24(1) $I_{i}^{\perp} \subseteq O(N)$. Therefore $d\left(a_{i}, a\right) \in O(N)$, for each $N \in U\left(b_{i}\right)$ and $i=1, \ldots, n$. It follows that $\left.\hat{a}\right|_{U\left(b_{i}\right)}=\hat{a}_{\left.i\right|_{U\left(b_{i}\right)}}=\sigma_{\mid U_{i}}$ for every $i=1,2, \ldots, n$, that is $\sigma=\hat{a}$.

Corollary 3.11 Every RMV-algebra is isomorphic to the algebra of global sections of a sheaf of local RMV-algebras over a compact Hausdorff space.

Proof By combining Theorem 3.8 with Lemma 3.10 .

\subsection{Spectral sheaf representation}

In this section we give an application of the following result by Cornish that provides sufficient conditions to represent an algebra using the spectrum of prime ideals as base space, topologised with the co-Zariski topology. Recall that the co-Zariski topology is defined on a set of prime ideals exactly as the Zariski topology, but the basic open sets and the basic closed sets are swapped. Recall also that a congruence $\theta$ is called prime, if $\theta=\theta_{1} \cap \theta_{2}$ implies $\theta=\theta_{1}$ or $\theta=\theta_{2}$. It is known, and easy to check, that prime congruences correspond to prime ideals by the isomorphisms of Lemma 2.14.

Theorem 3.12 (Cornish [11, Theorem 2.5]) Let $A$ be an algebra and $\mathcal{C}(A)$ be its set of congruences. Suppose that a subset $\mathcal{D} \subseteq \mathcal{C}(A)$ satisfies the following conditions:

(1) $\mathcal{D}$ contains the identity congruence id and the total congruence $\omega$.

(2) It is closed under intersections.

(3) $\mathcal{D}$ is upper regular in $\mathcal{C}(A)$, ie the join of any subset of $\mathcal{D}$ exists and coincide with the join in $\mathcal{C}(A)$.

(4) $\mathcal{D}$ is a distributive lattice.

(5) $\mathcal{D}$ consists of permuting congruences.

(6) $\mathcal{D}$ is compactly generated.

(7) The intersection of two compact elements in $\mathcal{D}$ is again compact.

Then $A$ is isomorphic to the sheaf associated to it as in Definition 3.4, where the space $(X, \tau)$ is the space of prime elements of $\mathcal{D}$ with the co-Zariski topology.

Notice that $\mathcal{D}$ satisfies (4) and (5) above if and only if it satisfies the Chinese Remainder Theorem (Lemma 2.28) relatively to the congruences in $\mathcal{D}$ (see [11, Lemma 2.2]).

Corollary 3.13 Every RMV-algebra $A$ is isomorphic to the algebra of global sections of a sheaf of linearly ordered $R M V$-algebras on the space $\operatorname{Spec}(A)$ with the co-Zariski topology. 
Proof Consider an RMV-algebra $A$ and take as $\mathcal{D}$ in Theorem 3.12 above the whole set of congruences on $A$. Then (1) to (3) and (6) are obvious. (4) and (5) hold because of Lemma 2.28 (although they are well-known to hold for MV-algebras, and the results obviously extend to RMV-algebras). Finally, to see that (7) holds, notice that the compact elements in the lattice of congruences of $A$ are the finitely-generated congruences, which correspond to principal ideals. So an application of Lemma 2.17 concludes the proof.

Remark 3.14 As one of the referees pointed out the two sheaf representations of this section could alternatively be derived by the recent results contained in Gehrke and van Gool [23] and in particular from Corollary 3.12 contained therein. Indeed, as noted after Theorem 3.12, the variety of RMV-algebras is both congruence permutable and congruence distributive. In addition RMV-algebras have the Compact Intersection Property, by Lemma 2.17. In contrast with the injectivity of Theorem 3.5(1), [23, Corollary 3.12] guarantees without further work that the algebra is isomorphic to the algebra of global sections of its representing sheaf, therefore our Lemma 3.10 would not be necessary. However, the results in [23] require extra work to characterise precisely the stalks in the representation, while in our approach they are clearly described by the construction itself.

\section{Local RMV-algebras}

\subsection{Localisation of RMV-algebras}

We begin this section motivating the name local RMV-algebras. The reasons are essentially similar to the ones that motivated the name local MV-algebra (see Belluce, Di Nola, and Gerla [2, Section 5]).

In modern terms one could define the abstract concept of localisation as follows.

Definition 4.1 Assume that $A$ is an algebraic structure in which it make sense to speak about prime and maximal ideal. Let $P$ be a prime ideal of $A$. A localization of $A$ at the prime $P$ is an algebra $B$ of the same type of $A$ such that:

(1) there exist a subalgebra of $A$ and a homomorphism $h$ such that $B$ is isomorphic to $h[A]$;

(2) the image of $P, h[P]$, is the unique maximal ideal of $B$; and

(3) $\operatorname{Spec}(B)$ is homeomorphic to the subspace of $\operatorname{Spec}(A)$ of prime ideals that are contained in $P$. 
Here by $\operatorname{Spec}(A)$ we mean the space of prime ideals of $A$ with the Zariski topology.

We will show that one can perform a localisation of an RMV-algebra at an arbitrary prime ideal. Unfortunately the localisation is not unique: this is the reason why we had to include the possibility of a subalgebra in (1) above; however, we will see that there is a canonical construction.

Thorough this section $A$ is assumed to be a non-trivial RMV-algebra. We fix some notation:

Notation Given an RMV-algebra $A$ and an ideal $P$ of $A$, we set

$$
\mathcal{L}_{A}(P):=\left\{A^{\prime} \leq A \mid P \text { is a maximal ideal of } A^{\prime}\right\}
$$

where $A^{\prime} \leq A$ means that $A^{\prime}$ is an RMV-subalgebra of $A$. We further set:

$$
\omega_{A}(P):=\{Q \in \operatorname{Spec}(A) \mid Q \subseteq P\} \text { and } \Omega_{A}(P):=\bigcap \omega_{A}(P)
$$

Finally, if $X \subseteq A$ we set $\operatorname{Ralg}(X)$ to be the RMV-subalgebra of $A$ generated by $X$.

Proposition 4.2 Let $P$ be any ideal of an $R M V$-algebra $A$. Then $P$ is a maximal ideal of $\operatorname{Ralg}(P)$. Hence $\operatorname{Ralg}(P) \in \mathcal{L}_{A}(P)$; in particular $\mathcal{L}_{A}(P) \neq \emptyset$.

Proof The support of the algebra $\operatorname{Ralg}(P)$ can be described as the set:

$$
\left\{t\left(p_{1}, \ldots, p_{k}\right) \mid t \text { is an RMV-term and } p_{1}, \ldots, p_{k} \in P\right\} \text {. }
$$

Recall that, by Lemma 2.14 , the ideal $P$ induces a congruence $\theta_{P}$. We prove by induction on the complexity of $t$ that every element $t\left(p_{1}, \ldots, p_{k}\right) \in \operatorname{Ralg}(P)$ is congruent modulo $\theta_{P}$ to an element of the form $f_{r}(1) \in[0,1]$. To simplify the induction steps, we use as basic operations $\ominus, 1$ and $\left\{f_{r} \mid r \in[0,1]\right\}$, justified by Remark 2.2. For the base step, it is readily seen that $1 \theta_{P} f_{1}(1)$ and $p \theta_{P} f_{1}(0)$ for every $p \in P$. For the inductive step first consider the element $t\left(p_{1}, \ldots, p_{k}\right) \ominus u\left(q_{1}, \ldots, q_{l}\right) \in \operatorname{Ral} g(P)$ for $t, u$ RMV-terms and $p_{1}, \ldots, p_{k}, q_{1}, \ldots, q_{l} \in P$. By induction hypothesis there exist $r, s \in[0,1]$ such that $t\left(p_{1}, \ldots, p_{k}\right) \theta_{P} f_{r}(1)$ and $u\left(q_{1}, \ldots, q_{l}\right) \theta_{P} f_{s}(1)$. Since $\theta_{P}$ is a congruence it also holds $t\left(p_{1}, \ldots, p_{k}\right) \ominus u\left(q_{1}, \ldots, q_{l}\right) \theta_{P} f_{r}(1) \ominus f_{s}(1)=f_{r \ominus s}(1)$, where the last equality is justified by (RMV 2). Similarly, if we consider the element $f_{\bar{r}}\left(t\left(p_{1}, \ldots, p_{k}\right)\right) \in \operatorname{Ralg}(P)$, then by induction hypothesis there exists $\bar{s} \in[0,1]$ such that $t\left(p_{1}, \ldots, p_{k}\right) \theta_{P} f_{\bar{s}}(1)$, hence $f_{\bar{r}}\left(t\left(p_{1}, \ldots, p_{k}\right)\right) \theta_{P} f_{\bar{r}}\left(f_{\bar{s}}(1)\right)=f_{\bar{r} \bar{S}}(1)$, where the last equality is justified by (RMV 3 ). This concludes the induction.

To prove that $P$ is maximal in $\operatorname{Ralg}(P)$ take an arbitrary $\bar{p} \in \operatorname{Ralg}(P) \backslash P$. Then there exists an $r \in[0,1]$ such that $\bar{p} \theta_{P} f_{r}(1)$, since $\bar{p} \notin P, f_{r}(1) \neq 0$. Since $f_{r}(1) \in \mathbb{R}(A)$ and 
$\mathbb{R}(A)$ is isomorphic to $[0,1]$, there is an $n \in \mathbb{N}$ such that $(n) f_{r}(1)=1$. Hence, $(n) \bar{p} \theta_{P} 1$, and this entails that $((n) \bar{p}))^{*} \theta_{P} 0$ hence $\left.((n) \bar{p})\right)^{*} \in P$. Since $(n) \bar{p} \oplus((n) \bar{p})^{*}=1$, we have that $P \cup\{\bar{p}\}$ generates the improper ideal in $\operatorname{Ralg}(P)$. In other words, $P$ is a maximal ideal of $\operatorname{Ral} g(P)$.

Note that $P$ is not necessarily the unique maximal ideal of $\operatorname{Ralg}(P)$. In fact, consider $A=[0,1]^{2}$ and $P$ the ideal generated by the element $(0,1)$. Then it is readily seen that $(0,1)$ generates the full RMV-algebra $A$, hence $\operatorname{Ralg}(P)=A$ but $A$ is obviously not local.

Proposition 4.3 Let $A$ be an $R M V$-algebra, $P$ any ideal of $A$, and $A^{\prime} \in \mathcal{L}_{A}(P)$. Then $A^{\prime} / \Omega_{A}(P)$ is a local $R M V$-algebra with maximal ideal $P / \Omega_{A}(P)$. In particular $\operatorname{Ral} g(P) / \Omega_{A}(P)$ is a local $R M V$-algebra with maximal ideal $P / \Omega_{A}(P)$.

Proof This was proved for MV-algebras in [2, Proposition 12]. Since it is a statement about ideals, in the light of Lemma 2.15, it also holds for RMV-algebras.

Theorem 4.4 Let $A$ be an $R M V$-algebra and $P$ a prime ideal of $A$. Then for any $A^{\prime} \in \mathcal{L}_{A}(P)$ there is a natural homeomorphism between $\omega_{A}(P)$ - seen as a subspace of $\operatorname{Spec}(A)$ with the Zariski topology-and $\operatorname{Spec}\left(A^{\prime} / \Omega_{A}(P)\right)$.

Proof This was proved for MV-algebras in [2, Proposition 15 and 16]. Since it is a statement about ideals, in the light of Lemma 2.15, it also holds for RMV-algebras.

Summing up, for every $A^{\prime} \in \mathcal{L}_{A}(P)$, and every $P \in \operatorname{Spec}(A)$, the algebra $A^{\prime} / O(P)$ satisfies Definition 4.1. In particular $\operatorname{Ral} g(P) / O(P)$ can be considered the canonical localization of the RMV-algebra $A$ at the prime ideal $P$.

The rest of this section is devoted to the study of local RMV-algebras.

\subsection{Quasi-constant functions}

Motivated by Theorem 2.29 we study RMV-algebras of $[0,1]^{*}$-valued functions, with $[0,1]^{*}$ an arbitrary non-principal ultrapower of the RMV-algebra $[0,1]$, and characterise local RMV-algebras among them.

Definition 4.5 If $[0,1]^{*}$ is any ultrapower of the RMV-algebra $[0,1]$, we call st (for standard part) the canonical quotient from $[0,1]^{*}$ into $[0,1]$ induced by (the congruence associated to) the ideal $\operatorname{Rad}\left([0,1]^{*}\right)$. 
Lemma 4.6 Let $[0,1]^{*}$ be an arbitrary ultrapower of the $R M V$-algebra $[0,1]$ and let $X$ be any set. The function st: $[0,1]^{*} \rightarrow[0,1]$ extends to a function $\tilde{\mathrm{st}}:\left([0,1]^{*}\right)^{X} \rightarrow$ $[0,1]^{X}$, defined for any $g \in\left([0,1]^{*}\right)^{X}$ by $\tilde{\text { st }}(g):=$ st $\circ g$, which is in turn induced by the ideal $\operatorname{Rad}\left(\left([0,1]^{*}\right)^{X}\right)$.

Proof Since st is surjective, st is surjective as well. Moreover we have:

$$
\begin{array}{rlr}
\operatorname{ker} & (\tilde{\mathrm{st}})=\left\{g \in\left([0,1]^{*}\right)^{X} \mid \tilde{\mathrm{st}}(g)=0\right\} & \text { by the definition of ker } \\
& =\left\{g \in\left([0,1]^{*}\right)^{X} \mid \operatorname{st}(g(x))=0 \text { for all } x \in X\right\} & \text { by the definition of st } \\
& =\left\{g \in\left([0,1]^{*}\right)^{X} \mid g(x) \in \operatorname{Rad}\left([0,1]^{*}\right) \text { for all } x \in X\right\} & \text { by the definition of st } \\
& =\left(\operatorname{Rad}\left([0,1]^{*}\right)\right)^{X} & \\
& =\operatorname{Rad}\left(\left([0,1]^{*}\right)^{X}\right) &
\end{array}
$$

So st is the quotient map of the MV-algebra $\left([0,1]^{*}\right)^{X}$ modulo the ideal $\operatorname{Rad}\left(\left([0,1]^{*}\right)^{X}\right)$.

Definition 4.7 If $A$ is an RMV-algebra and $x, y \in A$, we say that $x$ and $y$ are infinitely close if $\mathrm{d}(x, y) \in \operatorname{Rad}(A)$.

Definition 4.8 A function $g$ from a set $X$ into some non-trivial, linearly ordered RMV-algebra $C$ is called quasi-constant if it is infinitely close to some constant function; in symbols, if for all $x, y \in X, \mathrm{~d}(g(x), g(y)) \in \operatorname{Rad}(C)$.

Lemma 4.9 If $A$ is an $R M V$-algebra of quasi-constant functions into an ultrapower $[0,1]^{*}$ of the $R M V$-algebra on $[0,1]$, then $\tilde{\text { st }}[A] \cong[0,1]$.

Proof If $g \in A$ is quasi-constant, then $\tilde{\mathrm{st}}(\mathrm{g})$ is a constant function form $X$ into $[0,1]$, so $\tilde{\text { st }}[A]$ is embedded into $[0,1]$ by the map that evaluates functions in $\tilde{\text { st }}[A]$ at any point in $X$. Furthermore, by Theorem $2.11,[0,1]$ is a subalgebra of $\tilde{\mathrm{st}}[A]$. By Lemma 2.7, the composition of the two embedding must be the identity, hence $\tilde{\mathrm{st}}[A] \cong[0,1]$.

Theorem 4.10 An RMV-algebra is local if and only if it can be represented as an algebra of quasi-constant functions into an ultrapower $[0,1]^{*}$ of the RMV-algebra on $[0,1]$.

Proof Let $A$ be an RMV-algebra of quasi-constant functions from $X$ into some ultrapower $[0,1]^{*}$. An immediate application of Lemma 4.6 gives that the restriction 
$\tilde{\text { st }}$ to $A$ is induced by the ideal $\operatorname{Rad}(A)$. By Lemma $4.9, A / \operatorname{Rad}[0,1] \cong[0,1]$. Hence, by Lemma 2.22(1), $\operatorname{Rad}(A)$ is maximal, hence $A$ is local. For the other direction, let $A$ be a local RMV-algebra. Let $a \in A$ and $P \in \operatorname{Spec}(A)$, by Theorem 2.29 we identify $A / P$ with a suitable ultrapower $[0,1]^{*}$ so that $a$ corresponds to a function $g_{a}$ with values in $[0,1]^{*}$ such that $g_{a}(P)=[a]_{P}$ for every $P \in \operatorname{Spec}(A)$.

Let $M$ be the unique maximal ideal of $A$ and $P, P^{\prime} \in \operatorname{Spec}(A)$, then $A / P$ and $A / P^{\prime}$ are local and their unique maximal ideals are $M / P$ and $M / P^{\prime}$, respectively. Furthermore, by the second isomorphism theorem in Burris and Sankappanavar [6, Theorem 6.15, Chapter II] (together with the correspondence between congruences and ideals of Lemma 2.14), if $I \subseteq J$ are ideals of $A$, then there is an isomorphism from $\frac{A}{I} / \frac{J}{I}$ into $A / I$ that sends $\left[[a]_{I}\right]_{I / J}$ into $[a]_{J}$. Since by Lemma 2.22(1) the only endomorphism of $[0,1]$ is the identity we have:

$$
\operatorname{st}\left(g_{a}(P)\right)=\left[[a]_{P}\right]_{M / P}=[a]_{M}=\left[[a]_{P^{\prime}}\right]_{M / P^{\prime}}=\operatorname{st}\left(g_{a}\left(P^{\prime}\right)\right)
$$

Since $P$ and $P^{\prime}$ are arbitrary prime ideals, $g_{a}$ is quasi-constant, as required.

Corollary 4.11 An RMV-algebra is local if and only if it can be represented as an algebra of quasi-constant functions with values in a non-trivial, linearly ordered RMV-algebra.

Proof If an RMV-algebra $A$ is local, then by Theorem 4.10 it is an algebra of quasi-constant functions into an ultrapower $[0,1]^{*}$, and the latter is linearly ordered. Conversely, if $A$ can be represented as an algebra of quasi-constant functions with values in a linearly ordered RMV-algebra $C$, then by Theorem $2.29, C$ can be embedded into some ultrapower of $[0,1]$. Notice that the composition of a quasi-constant function into $C$ with this embedding gives a quasi-constant function with values in the ultrapower, because the image under the embedding of the radical of $C$ is included in the radical of the ultrapower. Hence we can again apply Theorem 4.10 to obtain that $A$ is local.

Remark 4.12 It is important to note that every non-trivial RMV-algebra $A$ has a largest local RMV-subalgebra. Indeed, by Theorem 2.29, $A$ can be represented as an algebra of functions from a set $I$ to an ultrapower $[0,1]^{*}$. Under this representation, the smallest RMV-subalgebra $\mathbb{R}(A)$ is given by the subalgebra of constant functions. Consider the set:

$$
L(A):=\{f \in A \mid f \text { is quasi-constant }\}
$$

The set $L(A)$ is obviously closed under all RMV-operations and contains all constant functions with range in $[0,1]^{*}$. So, by Theorem $4.10, L(A)$ is a local RMV-subalgebra 
of $A$. Take an arbitrary proper subalgebra $B$ of $A$ and suppose that $B$ is not contained in $L(A)$. Then, there is $b \in B$ which is not in $L(A)$. Then $b$ is (represented by) a function that is not quasi-constant. Hence, there must be $i, j \in I$ and two rationals $p<q$ such that $b(i)<p$ and $b(j)>q$. By McNaughton's Theorem [10, Theorem 9.1.5], one can find an MV-term $t$ such that $t(b)(i)=0$ and $t(b)(j)=1$. Let $a:=t(b) \in B$. Consider the ideal $J$ of $B$ given by all the functions in $B$ which are zero on $i: J$ is not contained in $\operatorname{Rad}(B)=\operatorname{Rad}(A) \cap B$ because $a \in J \backslash \operatorname{Rad}(B)$, so $B$ must have at least two distinct maximal ideals, hence it cannot be local.

\subsection{Radical retractions}

Lemma 4.13 Let $A$ be an $M V$-algebra and suppose $a, b \in A$ are infinitely close. Then:

(1) There exists $\varepsilon \in \operatorname{Rad}(A)$ such that $a \vee b=\varepsilon \oplus b$.

(2) The element a can be written as an $M V$-polynomial with coefficients in $\operatorname{Rad}(A) \cup$ $\{b\}$.

Proof (1). By hypothesis $(a \ominus b) \oplus(b \ominus a)=: \mathrm{d}(a, b) \in \operatorname{Rad}(A)$, since $\operatorname{Rad}(A)$ is an ideal, hence downward closed, this implies that also $(a \ominus b) \in \operatorname{Rad}(A)$. By definition $a \vee b:=(a \ominus b) \oplus b$, whence taking $\varepsilon:=(a \ominus b)$ settles the claim.

(2). If $a, b \in A$ are infinitely close then $a \ominus b, b \ominus a \in \operatorname{Rad}(A)$. Notice that:

$$
\begin{array}{rlr}
\mathrm{d}\left(a^{*},\right. & \left.a^{*} \wedge b^{*}\right) & \\
& =\left(a^{*} \ominus\left(a^{*} \wedge b^{*}\right)\right) \oplus\left(\left(a^{*} \wedge b^{*}\right) \ominus a^{*}\right) & \text { by Equation (3) } \\
& =\left(a^{*} \ominus\left(a^{*} \wedge b^{*}\right)\right) \oplus 0=a^{*} \ominus\left(a^{*} \wedge b^{*}\right) & \text { by Equation (5) and } a^{*} \wedge b^{*} \leq a^{*} \\
& =a^{*} \odot\left(a^{*} \wedge b^{*}\right)^{*}=a^{*} \odot(a \vee b) & \text { by Equation (3) and Equation (6) } \\
& =\left(a^{*} \odot a\right) \vee\left(a^{*} \odot b\right)=a^{*} \odot b & \text { by Proposition 2.5(1) and (5) } \\
& =b \ominus a \in \operatorname{Rad}(A) & \text { by Equation (3) }
\end{array}
$$

Therefore, $a^{*}$ and $a^{*} \wedge b^{*}$ are infinitely close. By absorption $a^{*}=\left(a^{*} \wedge b^{*}\right) \vee a^{*}$, hence we can apply (1) to obtain a $\tau \in \operatorname{Rad}(A)$ such that $a^{*}=\left(a^{*} \wedge b^{*}\right) \oplus \tau$. Now, by Equation (MV 3) and Equation (3) we obtain

$$
a=(a \vee b) \odot \tau^{*}=(\varepsilon \oplus b) \odot \tau^{*}
$$

where $\varepsilon, \tau \in \operatorname{Rad}(A)$, and this concludes the proof.

Theorem 4.14 An RMV-algebra $A$ is local if and only if it is generated by its radical. 
Proof If $A$ is generated by $\operatorname{Rad}(A)$, then, by Proposition 4.2, $\operatorname{Rad}(A)$ is maximal in $A$. Hence, since $\operatorname{Rad}(A)$ is contained in all maximal ideals, $\operatorname{Rad}(A)$ is the only maximal ideal of $A$ and $A$ is local.

Conversely, suppose that $A$ is local. By Theorem $4.10 A$ is isomorphic to an algebra of quasi-constant functions from a set $X$ into some ultrapower $[0,1]^{*}$. So, for any $a \in A$ there exists $r \in[0,1]$ such for all $i \in X$, st $(a(i))=r$. So, for all $i \in X, \mathrm{~d}\left(a(i), f_{r}(1)\right) \in \operatorname{Rad}\left([0,1]^{*}\right)$. Hence, by Lemma 4.13(2), $a$ can be written as a polynomial with coefficients in $\operatorname{Rad}(A) \cup b$, where $b \in \mathbb{R}(A)$. Since $\mathbb{R}(A)$ is the 0 -generated subalgebra of $A$, the algebra $A$ is generated by its radical.

Theorem 4.15 An RMV-algebra $A$ is local if and only if $A / \operatorname{Rad}(A) \cong[0,1]$.

Proof Preliminarily, notice that by Lemma $2.22(1), A / \operatorname{Rad}(A) \cong[0,1]$ if, and only if $\operatorname{Rad}(A)$ is maximal. Now, if $A$ is local, then $\operatorname{Rad}(A)$ is the only maximal ideal of $A$, therefore $A / \operatorname{Rad}(A) \cong[0,1]$. Vice versa, if $A / \operatorname{Rad}(A) \cong[0,1]$, then $\operatorname{Rad}(A)$ is maximal, whence $A$ is local.

Definition 4.16 Let $A$ be an RMV-algebra and $M$ be an ideal, we say that $A$ is $M$-retractive if there exists a section $s$ such that the following diagram commutes:

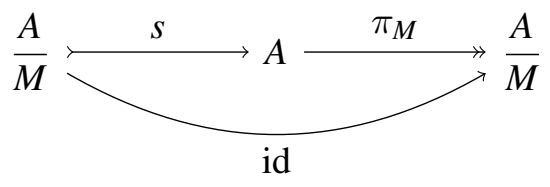

When this applies for $M=\operatorname{Rad}(A)$ we say that $A$ is Rad-retractive.

Proposition 4.17 Let $A$ be a non-trivial $R M V$-algebra and $M$ be a maximal ideal of $A$. Then $A$ is $M$-retractive and the section of the quotient map $A \rightarrow A / M$ is unique.

Proof The ideal $M$ is maximal, so by Lemma 2.22(1) there is an RMV-isomorphism $h: A / M \rightarrow[0,1]$. Let $\pi_{M}$ be the quotient map from $A$ to $A / M$. Let us define a map $j:[0,1] \rightarrow A$ stipulating that $j(x)=f_{x}(1)$. Now $\pi_{M} \circ j \circ h$ is an RMValgebra endomorphism of $A / M$; and since $A / M$ is isomorphic to [0,1], the only RMV endomorphism of $A / M$ is the identity (Lemma 2.7). So $\pi_{M} \circ j \circ h=i d$ and $j \circ h$ is a section of $\pi_{M}$.

To prove uniqueness, suppose $s, t$ are sections of $\pi_{M}$. Since $s, t$ factor the identity they must be injective, so $s(A / M)$ and $t(A / M)$ must be RMV-subalgebras of $A$, and they are 
both isomorphic to [0,1], by the maximality of $M$. So, by Theorem $2.11 s(A / M)=$ $t(A / M)=\mathbb{R}(A)$. Since, for every $a \in A, \pi_{M}(s(a / M))=\pi_{M}(t(a / M))=a / M$, the elements $s(a / M)$ and $t(a / M)$ must be constants congruent to $a$ modulo $M$. But, since $M$ is proper this can happen only if $s(a / M)=t(a / M)$. By the arbitrarily of $a$ we obtain $s=t$.

Remark 4.18 Notice that, as an immediate consequence of the previous proposition, every local RMV-algebra is Rad-retractive. Indeed, if $A$ is local, then $\operatorname{Rad}(A)$ is maximal.

Example 4.19 Consider the RMV-algebra $A$ obtained as direct product of two copies of the RMV-algebra $[0,1]$. Then:

(1) A is Rad-retractive. In fact, it is semisimple so the radical is zero, so trivially the algebra is radical-retractive (because it is zero-retractive).

(2) $A$ is not local. In fact, the ideal of pairs with the first component zero is maximal, and the one with the second component zero is also maximal. So $A$ shows that the implication of Remark 4.18 cannot be inverted.

(3) $A$ is retracts onto $[0,1]$. In fact, as a projection we can take (for instance) the projection of $A$ on the first component, and as a section the diagonal map sending $x \in[0,1]$ to $(x, x) \in A$. So $A$ shows that Theorem 4.15 cannot be trivially generalised.

One could conjecture that Rad-retractiveness is a property shared by all RMV-algebras. In fact, the conjecture is not easily disproved as all natural examples of RMV-algebras have this property. However, one can build a counter-example to the conjecture.

Proposition 4.20 There is an RMV-algebra which is not Rad-retractive.

Proof Let $[0,1]^{*}$ be the ultrapower of the RMV-algebra $[0,1]$ modulo a non-principal ultrafilter on $\mathbb{N}$. Let $\varepsilon \in \operatorname{Rad}\left([0,1]^{*}\right)$ and consider the function $g: \mathbb{N} \rightarrow[0,1]^{*}$ defined by $g(n)=\frac{1}{n}+\varepsilon$. Let $A$ be the RMV-algebra generated by $g$ in the RMV-algebra $\left([0,1]^{*}\right)^{\mathbb{N}}$. We will show that $A$ is not Rad-retractive.

We preliminarily show:

Claim 3 For every $a \in A$, if $a$ is infinitely close to $g$, then there is a unary $R M V$-term $u$ such that $u(a)>0$ and $u(a)$ is infinitesimal. 
Proof of Claim 3 Let $a \in A$. Since $A$ is generated by $g$ there exists a unary RMV-term $t$ such that $a=t(g)$. By Di Nola and Leuştean [18, Theorem 10] the interpretation of $t$ in the RMV-algebra [0,1] is a continuous piecewise linear function, let $\rho$ be its right derivative in 0 . Since there exists an open neighbourhood of 0 on which the interpretation of $t$ in $[0,1]$ is linear, $\rho$ is a real number and there exists $0 \neq m \in \mathbb{N}$ such that for any $n \geq m, t\left(\frac{1}{n}+\varepsilon\right)=\rho \cdot\left(\frac{1}{n}+\varepsilon\right)$. Since $t(g(n))$ is infinitely close to $g(n)$ for every $n \in \mathbb{N}$, it must be $\rho=1$. So,

$$
\text { for any } n \geq m, \quad t\left(\frac{1}{n}+\varepsilon\right)=\frac{1}{n}+\varepsilon .
$$

Set $u_{0}(x):=((m-1) x) \odot x$. Straightforward calculations show that Equation (11) implies that $u_{0}\left(t\left(\frac{1}{m}+\varepsilon\right)\right)$ is a positive infinitesimal, whereas for $n>m, u_{0}\left(t\left(\frac{1}{n}+\varepsilon\right)\right)=$ 0 . It follows that the set $I \subseteq \mathbb{N}$ such that for $i \in I, u_{0}\left(t\left(\frac{1}{i}+\varepsilon\right)\right)$ is not infinitesimal and different form 0 is finite. So there must exist $k \in \mathbb{N}$ such that $\frac{1}{k}<u_{0}\left(t\left(\frac{1}{i}+\varepsilon\right)\right)$ for all $i \in I$.

Set $u_{1}:=(k) u_{0}$. By previous calculations $u_{1}\left(t\left(\frac{1}{m}+\varepsilon\right)\right)=0$ is a positive infinitesimal, if $n>m$ then $u_{1}\left(t\left(\frac{1}{n}+\varepsilon\right)\right)=0$, and if $n<m$ then $u_{1}\left(t\left(\frac{1}{n}+\varepsilon\right)\right)$ is either equal to 1 or infinitesimal. Finally let $u(x):=\left(u_{1}(x)\right) \wedge\left(u_{1}(x)\right)^{*}$; we have $u\left(t\left(\frac{1}{n}+\varepsilon\right)\right)=0$ is a positive infinitesimal, if $n>m$ then $u\left(t\left(\frac{1}{n}+\varepsilon\right)\right)=0$, and if $n<m$ then $u\left(t\left(\frac{1}{n}+\varepsilon\right)\right)$ is either equal to 0 or infinitesimal. Summing up, $u(t(g))$ is a positive infinitesimal.

To conclude the proof of Proposition 4.20 we reason by way of contradiction. So, suppose that the quotient map $\pi: A \rightarrow A / \operatorname{Rad}(A)$ is a retraction, ie there is a section $s: A / \operatorname{Rad}(A) \rightarrow A$ such that $\pi \circ s$ is the identity. Notice that $s$ must be injective. Let $e:=s \circ \pi$, so $e \circ e=s \circ \pi \circ s \circ \pi=e$. The injectivity of $s$ amounts to saying that $e(x)=e(y)$ if and only if $\pi(x)=\pi(y)$, therefore form $e(x)=e(e(x))$ we deduce $\pi(x)=\pi(e(x))$; in other words $e(x)$ is infinitely close to $x$ for every $x \in A$ (intuitively, the map $e$ "chooses" a representative in each equivalence class modulo the radical). So, in particular, $e(g)$ is infinitely close to $g$, hence by Claim 3, there is a term $u$ such that $u(e(g))$ is infinitesimal and non-zero. It follows that $e(u(e(g)))=0$. Since $e$ is a homomorphism and $u$ is a term, we have $e(u(e(g)))=u(e(e(g)))=u(e(g)) \neq 0$ and this contradiction concludes the proof.

It should be noticed that an MV-algebra may be endowed with several non-isomorphic RMV-algebra structures, we refer the reader to Di Nola, Lenzi, Marra, and Spada [16] for more details on this and to Lenzi [28], which solves the corresponding problem posed by Conrad in 1975 . 
Remark 4.21 As an application of Corollary 3.11 one finds that if an MV-algebra $A$ is a reduct of an RMV-algebra, then $A$ must be isomorphic to the MV-algebra of global sections of a sheaf on a compact Hausdorff space, whose stalks are local MV-algebras that are radical retractive and whose quotient over their radical is isomorphic to $[0,1]$. Indeed, since the stalks are local RMV-algebras, they must be radical-retractive by Remark 4.18 and the quotient of each stalk modulo the radical is $[0,1]$ by Theorem 4.15. Obviously these properties are preserved under taking their MV-reducts.

Note that the converse does not hold. Indeed, consider a sheaf in which the base space is the one point topological space, and the unique stalk is given by an MV-subalgebra $A$ of $[0,1]^{*}$ generated by $[0,1] \cup\{\varepsilon\}$, where $\varepsilon$ is any positive infinitesimal. The MV-algebra of global sections of this sheaf is trivially isomorphic to $A$. The MV-algebra $A$ is local (being linearly ordered), $A / \operatorname{Rad}(A)=[0,1]$ (since every element of $A$ is infinitely close to an element of $[0,1])$ and $A$ is radical retractive (since $A / \operatorname{Rad}(A)=[0,1]$ embeds naturally in $A$ ), but $A$ is not a reduct of an RMV-algebra, because it is not even divisible. Indeed, every element of $A$ has the form $r \pm k \varepsilon$ where $r$ is real and $k$ is an integer, so the element $\varepsilon / 2$, which belongs to $[0,1]^{*}$, is not in $A$, hence $A$ is not even 2-divisible.

Theorem 4.22 Let $A$ be an $R M V$-algebra and let

$$
\rho_{\pi}: \prod_{P \in \operatorname{Spec}(A)} A / P \rightarrow \prod_{P \in \operatorname{Spec}(A)}(A / P) / \operatorname{Rad}(A / P)
$$

be the natural retraction whose components are given by the natural quotient maps $\pi_{\operatorname{Rad}(A / P)}$. The algebra $A$ is Rad-retractive if and only if $\rho_{\pi}(A)$ embeds in $A$.

Proof Suppose that $A$ is Rad-retractive and let $\rho$ be the section of the retraction, ie, $\pi_{\operatorname{Rad}(A)} \circ \rho=i d$. For every RMV-algebra $A$ there is a natural bijection $\beta$ between the RMV-algebras $\rho(A)$ and $\rho_{\pi}(A)$, such that $\beta(\rho(x))=\rho_{\pi}(x)$. In fact, we have $\rho(x)=\rho(y)$ if and only if $\rho_{\pi}(x)=\rho_{\pi}(y)$ if and only if $d(x, y)$ is infinitesimal, where $d(x, y)$ denotes Chang distance. Moreover, this bijection $\beta$ is an RMV-algebra homomorphism, hence $\rho_{\pi}(A)$ is isomorphic to $\rho(A)$ and embeds into $A$.

Conversely, if $\rho_{\pi}(A)$ embeds in $A$ via an embedding $j$, then the pair of maps $\left(j, \rho_{\pi}\right)$ is a retraction of $A$ on its radical quotient, and $A$ is Rad-retractive.

Note that a retraction on the factors of a direct product induces a retraction on the product, so every product of chains, finite or infinite, has a natural retraction by the previous lemma. 


\subsection{An equivalence between Local RMV-algebras and Riesz spaces}

Theorem 4.23 An RMV-algebra is local if and only if it is isomorphic to $\Gamma_{\mathcal{R}}(\mathbb{R} \vec{x}$ $\left.W,\left(1,0_{W}\right)\right)$ where $W$ is a Riesz space.

Proof Let $W$ be an arbitrary Riesz space and let $A \cong \Gamma_{R}\left(\mathbb{R} \overrightarrow{\times} W,\left(1,0_{W}\right)\right)$. We show that $A$ is local. Consider the set $M=\{a \in A \mid a=(0, w)$ for $w \in W\}$. It is easy to see that $M$ is an ideal of $A$. Furthermore, every element of $A \backslash M$ has the form $(r, w)$ with $0<r \leq 1$, so there exists a positive integer $n$ such that $r>1 / n$, whence $(n)(r, w)=1_{A}$. We conclude that no element of $A \backslash M$ can lie in a proper ideal of $A$. Summing up, $M$ is the greatest ideal of $A$, hence $A$ is local.

Conversely, let $A$ be a local RMV-algebra, hence by Theorem $4.14 A$ is generated by its radical. Now let $(V, u)$ be the Riesz space with order unit, given by Theorem 2.12, such that $A=\Gamma_{\mathcal{R}}(V, u)$. Then $V$, as a group, is generated by $A$. Moreover, since $A$ can be generated by $\operatorname{Rad}(A)$ by the RMV algebra operations, $A$ can also be generated by $\operatorname{Rad}(A)$ by using the Riesz space operations and $u$. Summing up, the Riesz space $V$ is generated by $\operatorname{Rad}(A) \cup\{u\}$. Let $W$ be the Riesz subspace of $V$ generated, as a Riesz space, by $\operatorname{Rad}(A)$. Note that every element $w$ of $W$ is infinitesimal with respect to $u$, in the sense that for every $n \in \mathbb{N}$ we have $n|w| \leq u$.

So, every element $v$ of $V$ can be written as $x u+w$, where $x$ is a real number and $w \in W$. The pair $(x, w)$ is unique, because if $x u+w=x^{\prime} u+w^{\prime}$ then $\left(x-x^{\prime}\right) u=w-w^{\prime}$, and the right hand side is infinitesimal with respect to $u$, whereas $u$ is not infinitesimal with respect to itself, so we must have $x-x^{\prime}=0$ and therefore $w=w^{\prime}$. The map $h$ sending $v$ to $(x, w)$ gives a map from $V$ to $\mathbb{R} \overrightarrow{\times} W$ which is a vector space isomorphism.

We are left with showing that $h$ is an isomorphism of ordered sets. For this it is enough to show that $x u+w \geq 0$ in $V$ if and only if $(x, w) \geq 0$ in $\mathbb{R} \overrightarrow{\times} W$. Suppose $x=0$. Then clearly $w \geq 0$ if and only if $(0, w) \geq 0$ in $\mathbb{R} \overrightarrow{\times} W$. Now suppose $x \neq 0$. Since $w$ is infinitesimal, we have $x u+w \geq 0$ in $V$ if and only if $x u \geq 0$ in $V$, and since $u \geq 0$, we have $x u \geq 0$ in $V$ if and only if $x \geq 0$. But $x \geq 0$ in $V$ if and only if $(x, w) \geq 0$ in $\mathbb{R} \overrightarrow{\times} W$. Summing up, $x u+w \geq 0$ in $V$ if and only if $(x, w) \geq 0$ in $\mathbb{R} \overrightarrow{\times} W$ as desired. 
Definition 4.24 Let

(1) LRMV be the category of local Riesz MV-algebras and RMV-homomorphisms,

(2) R be the category of Riesz spaces with Riesz homomorphisms, and

(3) $R_{1}$ be the category of Riesz spaces with order unit with Riesz homomorphisms preserving the order unit.

We define an assignment $\Delta^{0}: \mathrm{R} \rightarrow \mathrm{R}_{1}$ as follows.

(1) If $W$ is a Riesz space $\Delta^{0}(W):=\left(\mathbb{R} \overrightarrow{\times} W,\left(1,0_{W}\right)\right)$.

(2) If $h: W \rightarrow V$ is a homomorphism of Riesz spaces, $\Delta^{0}(h): \Delta^{0}(W) \rightarrow \Delta^{0}(V)$ is defined as the map $(r, w) \mapsto(r, h(w))$.

We further define $\Delta: \mathrm{R} \rightarrow \mathrm{LRMV}$ as $\Delta:=\Gamma_{\mathcal{R}} \circ \Delta^{0}$.

It is straightforward to check that $\Delta^{0}$, and hence $\Delta$, are functors.

Theorem 4.25 The functor $\Delta$ is faithful, full, and dense. Hence it gives a categorical equivalence between Riesz spaces and local RMV-algebras.

Proof To prove faithfulness it is enough to show that the functor $\Delta^{0}$ is faithful, since the composition of faithful functors is faithful. Now if $h, h^{\prime}: W \rightarrow V$ are two Riesz homomorphisms and $\Delta^{0}(h)=\Delta^{0}\left(h^{\prime}\right)$, then $(r, h(w))=\left(r, h^{\prime}(w)\right)$ for every $w \in W$, so $h=h^{\prime}$.

To prove fullness it is enough to show that the functor $\Delta^{0}$ is full, since the composition of full functors is full. Let $g:(\mathbb{R} \overrightarrow{\times} W,(1,0)) \rightarrow(\mathbb{R} \overrightarrow{\times} V,(1,0)$ be a Riesz morphism preserving the order unit. Then for every $r \in \mathbb{R}, g(r, 0)=r g(1,0)=r(1,0)=(r, 0)$. Since $g(r, 0)=(r, 0)$ for every $r \in \mathbb{R}$ and the map $g$ is increasing, the first component of $g(r, w)$ must be $r$ for every $w \in W$. Hence $g(r, w)=(r, k(r, w))$ for some function $k$. In particular $g(0, w)=(0, k(0, w))$, and

$g(r, w)=g(r, 0)+g(0, w)=g(r, 0)+(0, k(0, w))=(r, 0)+(0, k(0, w))=(r, k(0, w))$.

Notice that the function $h: W \rightarrow V$ defined as $h(w)=k(0, w)$ must be a homomorphism of Riesz spaces because $h(w)=\pi_{2} \circ g(0, w)$-where $\pi_{2}$ is the projection onto the second coordinate. So $g(r, w)=(r, h(w))=\Delta^{0}(k)$, whence $\Delta^{0}$ is full.

Density of $\Delta$ amounts to the fact that for every local RMV-algebra $A$ there exists a Riesz space $W$ such that $A \cong \Delta(W)$. This is given by the left-to-right implication of Theorem 4.23.

The equivalence is reminiscent of the equivalence between perfect MV-algebras and $\ell$-groups of Di Nola and Lettieri [17]. Note that in this case, perfect RMV-algebras do not exist, but in a sense they are replaced by local RMV-algebras. 


\section{References}

[1] C D Aliprantis, O Burkinshaw, Locally solid Riesz spaces with applications to economics, volume 105 of Mathematical Surveys and Monographs, second edition, American Mathematical Society, Providence, RI (2003); https://doi.org/10.1090/surv/105

[2] L P Belluce, A Di Nola, B Gerla, Perfect MV-algebras and their logic, Applied Categorical Structures 15 (2007) 135-151; https://doi.org/10.1007/s10485-007-9069-4

[3] I Ben Yaacov, A Usvyatsov, Continuous first order logic and local stability, Transactions of the American Mathematical Society 362 (2010) 5213-5259; https://doi.org/10.1090/S0002-9947-10-04837-3

[4] A Bigard, K Keimel, S Wolfenstein, Groupes et anneaux réticulés, volume 608, Springer (1977); https://doi.org/10.1007/BFb0067004

[5] G Birkhoff, Lattice Theory, volume 25 of American Mathematical Society Colloquium Publications, third edition, American Mathematical Society, New York, N. Y. (1973)

[6] S Burris, H P Sankappanavar, A course in universal algebra, volume 78 of Graduate Texts in Mathematics, Springer-Verlag, New York-Berlin (1981)

[7] L M Cabrer, L Spada, MV-algebras, infinite dimensional polyhedra, and natural dualities, Archive for Mathematical Logic 56 (2017) 21-42; https://doi.org/10.1007/s00153016-0512-9

[8] C C Chang, A new proof of the completeness of the Łukasiewicz axioms, Transactions of the American Mathematical Society 93 (1959) 74-80; https://doi.org/10.2307/1993423

[9] C-C Chang, H J Keisler, Continuous model theory, Annals of Mathematics Studies, No. 58, Princeton Univ. Press, Princeton, N.J. (1966)

[10] R L O Cignoli, I M L D'Ottaviano, D Mundici, Algebraic foundations of many-valued reasoning, volume 7 of Trends in Logic-Studia Logica Library, Kluwer Academic Publishers, Dordrecht (2000); https://doi.org/10.1007/978-94-015-9480-6

[11] W H Cornish, The Chinese remainder theorem and sheaf representations, Fundamenta Mathematicae 96 (1977) 177-187; https://doi.org/https://doi.org/10.4064/fm-96-3-177187

[12] B Davey, Sheaf spaces and sheaves of universal algebras, Mathematische Zeitschrift 134 (1973) 275-290; https://doi.org/10.1007/BF01214692

[13] A Di Nola, I Esposito, B Gerla, Local algebras in the representation of $M V$-algebras, Algebra Universalis 56 (2007) 133-164; https://doi.org/10.1007/s00012-007-1984-6

[14] A Di Nola, S Lapenta, I Leuştean, An analysis of the logic of Riesz spaces with strong unit, Annals of Pure and Applied Logic 169 (2018) 216-234; https://doi.org/10.1016/ j.apal.2017.10.006

[15] A Di Nola, S Lapenta, I Leuştean, Infinitary logic and basically disconnected compact Hausdorff spaces, Journal of Logic and Computation 28 (2018) 1275-1292; https://doi.org/10.1093/logcom/exy011 
[16] A Di Nola, G Lenzi, V Marra, L Spada, The algebras of piecewise-linear maps (2019); manuscript

[17] A Di Nola, A Lettieri, Perfect $M V$-algebras are categorically equivalent to abelian l-groups, Studia Logica 53 (1994) 417-432; https://doi.org/10.1007/BF01057937

[18] A Di Nola, I Leuştean, Eukasiewicz logic and Riesz spaces, Soft Computing 18 (2014) 2349-2363; https://doi.org/10.1007/s00500-014-1348-z

[19] E Dubuc, Y Poveda, Representation theory of $M V$-algebras, Annals of Pure and Applied Logic (2010) 1024-1046; https://doi.org/10.1016/j.apal.2009.12.006

[20] A R Ferraioli, Lukasiewicz logic: algebras and sheaves, $\mathrm{PhD}$ thesis, University of Salerno (2010)

[21] A Ferraioli, A Lettieri, Representations of $M V$-algebras by sheaves, Mathematical Logic Quarterly 57 (2011) 27-43; https://doi.org/10.1002/malq.200910116

[22] A Filipoiu, G Georgescu, Compact and Pierce representations of MV-algebras, Revue Roumaine de Mathématiques Pures et Appliquées 40 (1995) 599-618

[23] M Gehrke, S J v Gool, Sheaves and duality, Journal of Pure and Applied Algebra 222 (2018) 2164-2180; https://doi.org/10.1016/j.jpaa.2017.09.004

[24] M Gehrke, S J van Gool, V Marra, Sheaf representations of $M V$-algebras and lattice-ordered abelian groups via duality, Journal of Algebra 417 (2014) 290-332; https://doi.org/10.1016/j.jalgebra.2014.06.031

[25] L Gillman, M Jerison, Rings of continuous functions, Springer-Verlag, New YorkHeidelberg (1976). Reprint of the 1960 edition, Graduate Texts in Mathematics, No. 43

[26] C W Henson, Nonstandard hulls of Banach spaces, Israel Journal of Mathematics 25 (1976) 108-144; https://doi.org/10.1007/BF02756565

[27] J Iovino, Applications of model theory to functional analysis, Dover Publications, Inc., Mineola, NY (2014);

[28] G Lenzi, On the Riesz structures of a lattice ordered abelian group, Mathematica Slovaca 69 (2019) 1237-1244; https://doi.org/10.1515/ms-2017-0304

[29] W A J Luxemburg, A C Zaanen, Riesz spaces. Vol. I, North-Holland Mathematical Library, North-Holland Publishing Co. (1971)

[30] E Marchioni, Some notes on elimination properties for the theory of Riesz MV-chains, Mathematica Slovaca 65 (2015) 789-800; https://doi.org/10.1515/ms-2015-0055

[31] V Marra, L Spada, The dual adjunction between MV-algebras and Tychonoff spaces, Studia Logica 100 (2012) 253-278; https://doi.org/10.1007/s11225-012-9377-z

[32] D Mundici, Interpretation of AF $C^{*}$-algebras in Lukasiewicz sentential calculus, Journal of Functional Analysis 65 (1986) 15-63; https://doi.org/10.1016/0022-1236(86)90015-7 
[33] D Mundici, Advanced Łukasiewicz calculus and MV-algebras, volume 35 of Trends in Logic_Studia Logica Library, Springer, Dordrecht (2011); https://doi.org/10.1007/97894-007-0840-2

Dipartimento di Matematica, Università di Salerno. Piazza Caccioppoli, 284084 Fisciano (SA), Italy

Dipartimento di Matematica, Università di Salerno. Piazza Caccioppoli, 284084 Fisciano (SA), Italy

Dipartimento di Matematica, Università di Salerno. Piazza Caccioppoli, 284084 Fisciano (SA), Italy

adinola@unisa.it, gilenzi@unisa.it, Ispada@unisa.it

Received: 18 February 2020 Revised: 13 February 2021 